\title{
Untwisting the pure spinor formalism to the RNS and twistor string in a flat and $\operatorname{AdS}_{5} \times S^{5}$ background
}

\author{
Nathan Berkovits \\ ICTP South American Institute for Fundamental Research, \\ Instituto de Física Teórica, UNESP - Universidade Estadual Paulista, \\ Rua Dr. Bento T. Ferraz 271, 01140-070, São Paulo, SP, Brasil \\ E-mail: nberkovi@ift.unesp.br
}

ABSTRACT: The pure spinor formalism for the superstring can be formulated as a twisted $\mathrm{N}=2$ worldsheet theory with fermionic generators $j_{\mathrm{BRST}}$ and composite $b$ ghost. After untwisting the formalism to an $\mathrm{N}=1$ worldsheet theory with fermionic stress tensor $j_{\mathrm{BRST}}+$ $b$, the worldsheet variables combine into $\mathrm{N}=1$ worldsheet superfields $X^{m}$ and $\Theta^{\alpha}$ together with a superfield constraint relating $D X^{m}$ and $D \Theta^{\alpha}$. The constraint implies that the worldsheet superpartner of $\theta^{\alpha}$ is a bosonic twistor variable, and different solutions of the constraint give rise to the pure spinor or extended RNS formalisms, as well as a new twistor-string formalism with manifest $\mathrm{N}=1$ worldsheet supersymmetry.

These $\mathrm{N}=1$ worldsheet methods generalize in curved Ramond-Ramond backgrounds, and a manifestly $\mathrm{N}=1$ worldsheet supersymmetric action is proposed for the superstring in an $\mathrm{AdS}_{5} \times S^{5}$ background in terms of the twistor superfields. This $\mathrm{AdS}_{5} \times S^{5}$ worldsheet action is a remarkably simple fermionic coset model with manifest PSU $(2,2 \mid 4)$ symmetry and might be useful for computing $\mathrm{AdS}_{5} \times S^{5}$ superstring scattering amplitudes.

KeYwords: Superstrings and Heterotic Strings, AdS-CFT Correspondence, Topological Strings

ARXIV EPRINT: 1604.04617

Dedicated to the memory of Mario Tonin. 


\section{Contents}

1 Introduction 1

2 Untwisting the pure spinor formalism 4

$2.1 \mathrm{~N}=1$ generators and superfields 4

$\begin{array}{ll}2.2 \text { Worldsheet supersymmetric action } & 6\end{array}$

$2.3 \mathrm{U}(1)$ generator 8

2.4 Massless vertex operators 9

$\begin{array}{ll}2.5 & \text { Tree-level scattering amplitudes } \\ & 10\end{array}$

2.6 Extended RNS formalism 11

2.7 Worldsheet supersymmetric action in curved background 13

3 Twistor string formalism $\quad \mathbf{1 5}$

$\begin{array}{ll}3.1 \text { Twistor superfields } & 15\end{array}$

$\begin{array}{ll}3.2 \mathrm{~N}=1 \text { superconformal and } \mathrm{U}(1) \text { generator } & 17\end{array}$

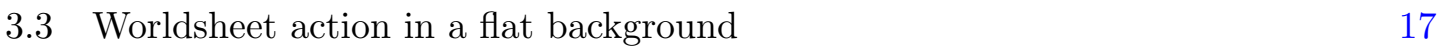

3.4 Twistor string in $\mathrm{AdS}_{5} \times S^{5}$ background 20

$3.5 \mathrm{U}(1)$ generator and $\mathrm{d}=12$ pure spinors 22

\section{Introduction}

The pure spinor formalism for the superstring [1] has the advantage over the RamondNeveu-Schwarz (RNS) formalism in that is manifestly spacetime supersymmetric. This simplifies the computation of multiloop superstring amplitudes [2] since there is no sum over spin structures, and allows the description of Ramond-Ramond superstring backgrounds such as $\mathrm{AdS}_{5} \times S^{5}[3]$. However, the pure spinor formalism has the disadvantage that it is not manifestly worldsheet supersymmetric. This complicates the construction of the $b$ ghost and integrated vertex operators, and introduces subtleties associated with regulators [4] and contact terms [5] needed to preserve BRST invariance.

Although the pure spinor formalism is not manifestly worldsheet supersymmetric, it has a twisted $\mathrm{N}=2$ worldsheet supersymmetry in which the two fermionic $\mathrm{N}=2$ generators are the BRST current and the $b$ ghost [6]. In this paper, the $\mathrm{N}=2$ worldsheet supersymmetry will be untwisted and the pure spinor formalism will be described in a manifestly $\mathrm{N}=1$ worldsheet supersymmetric and $\mathrm{d}=10$ spacetime supersymmetric manner in terms of the $\mathrm{N}=1$ worldsheet superfields

$$
X^{m}=x^{m}+\kappa \psi^{m}, \quad \Theta^{\alpha}=\theta^{\alpha}+\kappa \Lambda^{\alpha}, \quad \Phi_{\alpha}=\Omega_{\alpha}+\kappa h_{\alpha},
$$

where $\kappa$ is the anticommuting coordinate, $\left(x^{m}, \theta^{\alpha}\right)$ are the usual $\mathrm{d}=10$ superspace variables, $\left(\psi^{m}, \Lambda^{\alpha}\right)$ are their worldsheet superpartners, and $\left(\Omega_{\alpha}, h_{\alpha}\right)$ are the conjugate momenta to $\left(\Lambda^{\alpha}, \theta^{\alpha}\right)$. 
The $\mathrm{N}=1$ worldsheet superfields of (1.1) are constrained to satisfy

$$
\bar{\lambda} \gamma^{m} \Phi=0, \quad\left(\bar{\lambda} \gamma^{m}\right)_{\alpha}\left(D X_{m}-\frac{1}{2} D \Theta \gamma_{m} \Theta\right)=0
$$

where $\bar{\lambda}_{\alpha}$ is a fixed $d=10$ pure spinor satisfying $\bar{\lambda} \gamma^{m} \bar{\lambda}=0$. Although the constraints of (1.2) break manifest Lorentz covariance, one can solve these constraints using three different methods to produce three different Lorentz-covariant descriptions of the superstring.

The first method is to solve for $\psi^{m}$ and $\Lambda^{\alpha}$ in terms of $\left(x^{m}, \theta^{\alpha}, \lambda^{\alpha}\right)$ where $\lambda^{\alpha}$ is a $\mathrm{d}=10$ pure spinor satisfying $\lambda \gamma^{m} \lambda=0$. This method produces the pure spinor formalism which is manifestly spacetime supersymmetric but not manifestly worldsheet supersymmetric, and where the $\mathrm{N}=1$ fermionic generator is the sum of the pure spinor BRST current and $b$ ghost.

The second method is to solve for $\theta^{\alpha}$ and $\Lambda^{\alpha}$ in terms of $\left(x^{m}, \psi^{m}, \theta^{\prime \alpha}, \lambda^{\alpha}\right)$ where $\theta^{\prime \alpha}$ is constrained to satisfy $\theta^{\prime} \gamma^{m} \lambda=0$ and $\lambda^{\alpha}$ is constrained to satisfy $\lambda \gamma^{m} \lambda=0$. This method is manifestly worldsheet supersymmetric where $\lambda^{\alpha}$ is the worldsheet superpartner of $\theta^{\prime \alpha}$, but is not manifestly spacetime supersymmetric. One can argue that $\left(\theta^{\prime \alpha}, \lambda^{\alpha}\right)$ decouples from physical vertex operators and scattering amplitudes, so this method produces an "extended" version of the RNS formalism where $X^{m}=x^{m}+\kappa \psi^{m}$ plays the role of the usual RNS matter superfield.

Finally, the third method is to solve for $x^{m}$ and $\psi^{m}$ in terms of $\left(\Lambda^{\alpha}, \theta^{\alpha}\right)$ and its conjugate momenta $\left(\Omega_{\alpha}, h_{\alpha}\right)$. This method preserves both manifest worldsheet supersymmetry and spacetime supersymmetry, and produces a twistor description of the superstring in which $\left(\Lambda^{\alpha}, \Omega_{\alpha}\right)$ are $\mathrm{d}=10$ twistor variables which replace the $x^{m}$ spacetime variable.

There are several similarities of this worldsheet supersymmetric twistor description with earlier twistor descriptions of the superstring in [7-14], however, these earlier twistor descriptions were mostly for the heterotic superstring whereas this twistor description is only for the Type II superstring. It would be very interesting to study the relation of these twistor descriptions to each other, as well as to the more recent twistor superstrings which describe either $\mathrm{N}=4 \mathrm{~d}=4$ super-Yang-Mills $[15,16]$ or $\mathrm{d}=10$ supergravity [17, 18].

In a flat background, the $\mathrm{N}=(1,1)$ worldsheet supersymmetric action for the Type II twistor superstring is

$$
S=\int d^{2} z d^{2} \kappa\left[-\Phi_{\alpha} \bar{D} \Theta^{\alpha}+\widehat{\Phi}_{\hat{\alpha}} D \widehat{\Theta}^{\hat{\alpha}}-\frac{1}{8}\left(\Theta \gamma^{m} D \Theta\right)\left(\widehat{\Theta} \gamma_{m} \bar{D} \widehat{\Theta}\right)+\frac{1}{8}\left(\widehat{\Theta} \gamma^{m} D \widehat{\Theta}\right)\left(\Theta \gamma_{m} \bar{D} \Theta\right)\right]
$$

where $D=\frac{\partial}{\partial \kappa}+\kappa \partial_{z}$ and $\bar{D}=\frac{\partial}{\partial \bar{\kappa}}+\bar{\kappa} \bar{\partial}_{\bar{z}},\left(\Theta^{\alpha}, \Phi_{\alpha}, \widehat{\Theta}^{\hat{\alpha}}, \widehat{\Phi}_{\hat{\alpha}}\right)$ are $\mathrm{N}=(1,1)$ worldsheet superfields and $\alpha, \hat{\alpha}=1$ to 16 are $\mathrm{d}=10$ spinor indices of the same/opposite chirality for the Type IIB/IIA superstring. This action is manifestly invariant under both $\mathrm{N}=(1,1)$ worldsheet supersymmetry and $d=10 \mathrm{~N}=2$ spacetime supersymmetry which transforms the worldsheet superfields as

$$
\begin{array}{ll}
\delta \Theta^{\alpha}=\epsilon^{\alpha}, & \delta \widehat{\Theta}^{\hat{\alpha}}=\widehat{\epsilon}^{\hat{\alpha}}, \\
\delta \Phi_{\alpha}=\frac{1}{4}\left(\epsilon \gamma^{m} \Theta+\widehat{\epsilon} \gamma^{m} \widehat{\Theta}\right)\left(\gamma_{m} D \Theta\right), & \delta \widehat{\Phi}_{\hat{\alpha}}=\frac{1}{4}\left(\epsilon \gamma^{m} \Theta+\widehat{\epsilon} \gamma^{m} \widehat{\Theta}\right)\left(\gamma_{m} \bar{D} \widehat{\Theta}\right) .
\end{array}
$$


Surprisingly, when expressed in terms of these twistor superfields, the Type IIB superstring action in an $\mathrm{AdS}_{5} \times S^{5}$ background takes the extremely simple form

$$
S=r^{2} \int d^{2} z d^{2} \kappa\left[D \Theta_{R}^{J} \bar{D} \tilde{\Theta}_{J}^{R}+D \Theta_{S}^{J} \tilde{\Theta}_{K}^{S} \bar{D} \Theta_{R}^{K} \tilde{\Theta}_{J}^{R}\right]
$$

where $r$ is the AdS radius, $R=1$ to 4 are $\mathrm{SO}(4,2)$ spinor indices for $\operatorname{AdS}_{5}, J=1$ to 4 are $\mathrm{SO}(6)$ spinor indices for $S^{5}$, the 16 components of the superfield $\Theta_{R}^{J}$ are obtained by decomposing $\Theta^{\alpha}+i \widehat{\Theta}^{\hat{\alpha}}$ under $\mathrm{SO}(4,2) \times \mathrm{SO}(6)$, and the 16 components of the superfield $\tilde{\Theta}_{J}^{R}$ are obtained by decomposing $\Theta^{\alpha}-i \widehat{\Theta}^{\hat{\alpha}}$. This $\mathrm{AdS}_{5} \times S^{5}$ twistor-string action is manifestly invariant under both $\mathrm{N}=(1,1)$ worldsheet supersymmetry and under $\operatorname{PSU}(2,2 \mid 4)$ where the 32 spacetime supersymmetries transform the worldsheet superfields as

$$
\delta \Theta_{R}^{J}=\epsilon_{R}^{J}+\Theta_{S}^{J} \tilde{\epsilon}_{K}^{S} \Theta_{R}^{K}, \quad \delta \tilde{\Theta}_{J}^{R}=\tilde{\epsilon}_{J}^{R}-\tilde{\epsilon}_{K}^{R} \Theta_{S}^{K} \tilde{\Theta}_{J}^{S}-\tilde{\Theta}_{K}^{R} \Theta_{S}^{K} \tilde{\epsilon}_{J}^{S} .
$$

Hopefully, the simple form of (1.5) will be useful for constructing vertex operators and computing superstring scattering amplitudes in an $\mathrm{AdS}_{5} \times S^{5}$ background. But before constructing vertex operators and computing scattering amplitudes using this twistor-string action in an $\mathrm{AdS} \times S^{5}$ background, it will be necessary to better understand the vertex operators and scattering amplitudes using the twistor-string action in a flat background of (1.3).

In section 2.1 , the $\mathrm{N}=2$ worldsheet supersymmetry of the pure spinor formalism is untwisted to an $\mathrm{N}=1$ worldsheet supersymmetry with the fermionic generator $G=j_{\mathrm{BRST}}+b$, and the constrained $\mathrm{N}=1$ worldsheet superfields $\left[X^{m}, \Theta^{\alpha}, \Phi_{\alpha}\right]$ satisfying (1.2) are defined. In section 2.2 , the $\mathrm{N}=1$ worldsheet supersymmetric action with manifest $\mathrm{d}=10$ supersymmetry is constructed for the superstring in a flat background in terms of these constrained superfields. In section 2.3, the $\mathrm{U}(1)$ generator $J=-\lambda^{\alpha} w_{\alpha}$ is used to define physical states whose integrated vertex operator is required to be $\mathrm{N}=1$ superconformally invariant and have zero or negative $\mathrm{U}(1)$ charge. In section 2.4, physical vertex operators are constructed for massless states and the Siegel gauge-fixing condition $b_{0}=0$ is clarified. In section 2.5, a new tree amplitude prescription is given for the pure spinor formalism based on the untwisted approach which matches the RNS tree amplitude prescription in the $\mathcal{F}_{1}$ picture. In section 2.6, an alternative solution to the superfield constraints of (1.2) is shown to produce an extended version of the RNS formalism where the $\left[\Theta^{\alpha}, \Phi_{\alpha}\right]$ superfields decouple from the $X^{m}$ superfield. And in section 2.7, the $\mathrm{N}=1$ worldsheet supersymmetric approach to the pure spinor formalism is generalized to curved heterotic and Type II supergravity backgrounds.

In section 3.1, a third solution to the superfield constraints of (1.2) for the Type II superstring is described which replaces the usual spacetime variable $x^{m}$ with twistor variables and solves for $X^{m}$ in terms of $\left[\Theta^{\alpha}, \Phi_{\alpha}\right]$. In section $3.2, \mathrm{~N}=1$ worldsheet superconformal generators are constructed for this Type II twistor-string formalism and a U(1) generator corresponding to the projective weight of $\mathrm{d}=10$ twistors is used to define physical states. In section 3.3 , the $\mathrm{N}=(1,1)$ worldsheet supersymmetric twistor-string action in a flat background of (1.3) is constructed and shown to be equivalent to the usual pure spinor Type II superstring action up to a BRST-trivial term. In section 3.4, this twistor-string action is generalized in an $\mathrm{AdS}_{5} \times S^{5}$ background to the remarkably simple action of (1.5) which 
has manifest $\operatorname{PSU}(2,2 \mid 4)$ symmetry and reduces in the large radius limit to the action in a flat background of (1.3). Finally, in section 3.5, the $\mathrm{AdS}_{5} \times S^{5}$ twisor-string action is written in $\mathrm{SO}(10,2)$ notation and a $\mathrm{U}(1)$ generator involving $\mathrm{d}=12$ pure spinors is used to define physical states.

\section{Untwisting the pure spinor formalism}

\section{1 $\mathrm{N}=1$ generators and superfields}

In a flat background, the left-moving variables of the pure spinor formalism for the superstring are described in conformal gauge by the free worldsheet action

$$
S=\int d^{2} z\left(\frac{1}{2} \partial x^{m} \bar{\partial} x_{m}+p_{\alpha} \bar{\partial} \theta^{\alpha}+w_{\alpha} \bar{\partial} \lambda^{\alpha}\right),
$$

where $\left(x^{m}, \theta^{\alpha}\right)$ are the usual $\mathrm{N}=1 \mathrm{~d}=10$ superspace variables for $m=0$ to 9 and $\alpha=1$ to $16, p_{\alpha}$ is the conjugate momenta to $\theta^{\alpha}, \lambda^{\alpha}$ is a $\mathrm{d}=10$ pure spinor variable satisfying $\lambda \gamma^{m} \lambda=0$, and $w_{\alpha}$ is the conjugate momentum to $\lambda^{\alpha}$ which is defined up to the gauge transformation $\delta w_{\alpha}=f^{m}\left(\gamma_{m} \lambda\right)_{\alpha}$.

As discussed in [6], this pure spinor formalism can be interpreted as a topologically twisted $\mathrm{N}=2$ worldsheet superconformal field theory with fermionic left-moving generators

$$
\begin{aligned}
& G^{+}=j_{\mathrm{BRST}}=\lambda^{\alpha} d_{\alpha} \\
& G^{-}=b=-w_{\alpha} \partial \theta^{\alpha}+\frac{1}{2(\lambda \bar{\lambda})}\left[\pi^{m}\left(\bar{\lambda} \gamma_{m} d\right)+\left(w \gamma_{m} \bar{\lambda}\right)\left(\lambda \gamma^{m} \partial \theta\right)\right]
\end{aligned}
$$

where $\oint G^{+}$is the BRST charge used to define physical states, $G^{-}$is the composite $b$ ghost used for computing loop amplitudes, $\bar{\lambda}_{\alpha}$ is a fixed pure spinor on a patch defined by $\left(\lambda^{\alpha} \bar{\lambda}_{\alpha}\right) \neq 0$, and $\pi^{m}$ and $d_{\alpha}$ are the spacetime supersymmetric operators

$$
\pi^{m}=\partial x^{m}-\frac{1}{2} \partial \theta \gamma^{m} \theta, \quad d_{\alpha}=p_{\alpha}-\frac{1}{2} \partial x_{m}\left(\gamma^{m} \theta\right)_{\alpha}-\frac{1}{8}\left(\theta \gamma^{m} \partial \theta\right)\left(\gamma_{m} \theta\right)_{\alpha} .
$$

Using the OPE's from the free worldsheet action of (2.1), one can verify that $G^{+}$and $G^{-}$ are nilpotent operators satisfying the relation

$$
\left\{\oint G^{+}, G^{-}\right\}=T_{\text {twisted }}=-\frac{1}{2} \partial x^{m} \partial x_{m}-p_{\alpha} \partial \theta^{\alpha}-w_{\alpha} \partial \lambda^{\alpha}
$$

for any choice of $\bar{\lambda}_{\alpha}$. Although $G^{-}$can be Lorentz-covariantized by treating $\bar{\lambda}_{\alpha}$ as a nonminimal worldsheet variable [6], this non-minimal version of the pure spinor formalism will not be discussed here and $\bar{\lambda}_{\alpha}$ will be assumed to be fixed on each patch. Furthermore, we will be ignoring all normal-ordering terms and central charges thoughout this paper such as the term proportional to $\bar{\lambda}_{\alpha} \partial^{2} \theta^{\alpha}$ in $G^{-}$. Hopefully, the non-minimal formalism and normal-ordering contributions will be treated in a later paper.

To untwist the $\mathrm{N}=2$ generators of (2.2), define the $\mathrm{N}=1$ generator

$$
G=G^{+}+G^{-}=\lambda^{\alpha} d_{\alpha}-w_{\alpha} \partial \theta^{\alpha}+\frac{1}{2(\lambda \bar{\lambda})}\left[\pi^{m}\left(\bar{\lambda} \gamma_{m} d\right)+\left(w \gamma_{m} \bar{\lambda}\right)\left(\lambda \gamma^{m} \partial \theta\right)\right]
$$


which satisfies the OPE of an $\mathrm{N}=1$ superconformal stress tensor

$$
G(y) G(z) \rightarrow 2(y-z)^{-1} T(z)
$$

where

$$
T=-\frac{1}{2} \partial x^{m} \partial x_{m}-p_{\alpha} \partial \theta^{\alpha}-\frac{1}{2}\left(w_{\alpha} \partial \lambda^{\alpha}-\lambda^{\alpha} \partial w_{\alpha}\right)
$$

is the untwisted stress tensor with $\left(\lambda^{\alpha}, w_{\alpha}\right)$ of $+\frac{1}{2}$ conformal weight, and the central charge contribution in (2.6) is being ignored.

Under the $\mathrm{N}=1$ worldsheet supersymmetry generated by $G$ of $(2.5)$, the bosonic worldsheet superpartner $G \theta^{\alpha}$ of $\theta^{\alpha}$ is

$$
\Lambda^{\alpha}=\lambda^{\alpha}+\frac{1}{2(\lambda \bar{\lambda})} \pi^{m}\left(\gamma_{m} \bar{\lambda}\right)^{\alpha}
$$

and the fermionic worldsheet superpartner $G x^{m}$ of $x^{m}$ is

$$
\psi^{m}=\frac{1}{2} \Lambda \gamma^{m} \theta-\frac{1}{2(\lambda \bar{\lambda})}\left(\bar{\lambda} \gamma^{m} d\right) .
$$

So one can define $\mathrm{N}=1$ worldsheet superfields

$$
X^{m}=x^{m}+\kappa \psi^{m}, \quad \Theta^{\alpha}=\theta^{\alpha}+\kappa \Lambda^{\alpha}
$$

where $\kappa$ is an anticommuting parameter, which transform covariantly under $\mathrm{N}=1$ worldsheet supersymmetry transformations and transform under the $\mathrm{d}=10$ spacetime supersymmetry transformations as $\delta \Theta^{\alpha}=\epsilon^{\alpha}, \delta X^{m}=-\frac{1}{2} \epsilon \gamma^{m} \Theta$.

Furthermore, the conjugate momenta variables $p_{\alpha}$ and $w_{\alpha}$ can be combined into the worldsheet superfield

$$
\Phi_{\alpha}=w_{\alpha}-\frac{1}{2(\lambda \bar{\lambda})}\left(w \gamma_{m} \bar{\lambda}\right)\left(\gamma^{m} \lambda\right)+\kappa\left[d_{\alpha}-\frac{1}{2(\lambda \bar{\lambda})}\left(d \gamma_{m} \bar{\lambda}\right)\left(\gamma^{m} \lambda\right)_{\alpha}-\frac{1}{2(\lambda \bar{\lambda})^{2}} \bar{\lambda}_{\alpha}\left(\bar{\lambda} \gamma_{m} d\right) \pi^{m}\right]
$$

where $\pi^{m}$ and $d_{\alpha}$ are defined in (2.3). Note that $\Phi_{\alpha}$ has conformal weight $+\frac{1}{2}$ and transforms covariantly under $\mathrm{N}=1$ worldsheet supersymmetry, and is spacetime supersymmetric. The $\mathrm{N}=1$ superfields $\left(X^{m}, \Theta^{\alpha}, \Phi_{\alpha}\right)$ are not independent and satisfy the worldsheet and spacetime supersymmetric constraints

$$
\left(\gamma^{m} \bar{\lambda}\right)^{\alpha}\left(D X_{m}-\frac{1}{2} D \Theta \gamma_{m} \Theta\right)=0, \quad\left(\bar{\lambda} \gamma^{m}\right)^{\alpha} \Phi_{\alpha}=0
$$

where $D=\frac{\partial}{\partial \kappa}+\kappa \frac{\partial}{\partial z}$.

As will be shown later, different solutions of the constraints of (2.12) will describe either the pure spinor formalism, an extended version of the RNS formalism, or a new twistor formalism of the superstring. 


\subsection{Worldsheet supersymmetric action}

To construct the $\mathrm{N}=(1,0)$ worldsheet supersymmetric action for the heterotic superstring, generalize the superfields of (2.10) and (2.11) to the off-shell $N=(1,0)$ superfields

$$
X^{m}=x^{m}+\kappa \psi^{m}, \quad \Theta^{\alpha}=\theta^{\alpha}+\kappa \Lambda^{\alpha}, \quad \Phi_{\alpha}=\Omega_{\alpha}+\kappa h_{\alpha},
$$

where $\left(x^{m}, \psi^{m}, \theta^{\alpha}, \Lambda^{\alpha}, \Omega_{\alpha}, h_{\alpha}\right)$ are treated as independent components. For the heterotic superstring in a flat background, the $\mathrm{N}=(1,0)$ worldsheet action in terms of these superfields is

$$
S=\int d^{2} z d \kappa\left[\Phi_{\alpha} \bar{\partial} \Theta^{\alpha}+\frac{1}{2} \Pi_{\kappa}^{m} \bar{\Pi}_{\bar{z} m}+B_{\kappa \bar{z}}^{\mathrm{het}}+\left(\bar{\lambda} \gamma^{m} L\right) \Pi_{\kappa m}+M^{m}\left(\bar{\lambda} \gamma_{m} \Phi\right)\right]
$$

where

$$
\begin{aligned}
\Pi_{\kappa}^{m} & =D X^{m}-\frac{1}{2} D \Theta \gamma^{m} \Theta, \quad \Pi_{z}^{m}=\partial X^{m}-\frac{1}{2} \partial \Theta \gamma^{m} \Theta, \quad \bar{\Pi}_{\bar{z}}^{m}=\bar{\partial} X^{m}-\frac{1}{2} \bar{\partial} \Theta \gamma^{m} \Theta, \\
B_{\kappa \bar{z}}^{\text {het }} & =\frac{1}{4}\left[\left(D \Theta \gamma_{m} \Theta\right) \bar{\partial} X^{m}-D X^{m}\left(\bar{\partial} \Theta \gamma_{m} \Theta\right)\right], \quad B_{z \bar{z}}^{\text {het }}=\frac{1}{4}\left[\left(\partial \Theta \gamma_{m} \Theta\right) \bar{\partial} X^{m}-\partial X^{m}\left(\bar{\partial} \Theta \gamma_{m} \Theta\right)\right],
\end{aligned}
$$

$B_{z \bar{z}}^{\text {het }}$ is the usual heterotic Green-Schwarz two-form, $B_{\kappa \bar{z}}^{\text {het }}$ is obtained from $B_{z \bar{z}}^{\text {het }}$ by replacing $\partial_{z}$ with $D, L_{\alpha}$ and $M^{m}$ are Lagrange multiplier superfields enforcing the constraints of (2.12), and the right-moving fermions of the heterotic superstring [19] which generate the $\mathrm{SO}(32)$ or $E_{8} \times E^{8}$ gauge groups will be ignored throughout this paper.

Performing the Grassmann integral over $\kappa$ and imposing the constraints of (2.12), the action of (2.14) is equal to

$$
\begin{aligned}
S= & \int d^{2} z\left[D \Phi_{\alpha} \bar{\partial} \Theta^{\alpha}+\Phi_{\alpha} \bar{\partial} D \Theta^{\alpha}+\frac{1}{2}\left(\Pi_{z}^{m}-\frac{1}{2} D \Theta \gamma^{m} D \Theta\right) \bar{\Pi}_{\bar{z} m}-\frac{1}{2} \Pi_{\kappa m}\left(\bar{\partial} \Pi_{\kappa}^{m}+D \Theta \gamma^{m} \bar{\partial} \Theta\right)\right. \\
& \left.+B_{z \bar{z}}^{\text {het }}+\frac{1}{2}\left(D \Theta \gamma^{m} \bar{\partial} \Theta\right) \Pi_{\kappa}+\frac{1}{4}\left(D \Theta \gamma^{m} D \Theta\right) \bar{\Pi}_{\bar{z}}\right] \\
= & \int d^{2} z\left[D \Phi_{\alpha} \bar{\partial} \Theta^{\alpha}+\Phi_{\alpha} \bar{\partial} D \Theta^{\alpha}+\frac{1}{2} \Pi_{z}^{m} \bar{\Pi}_{\bar{z} m}-\Pi_{\kappa}^{m} D \Theta \gamma_{m} \bar{\partial} \Theta+B_{z \bar{z}}^{\text {het }}\right] \\
= & \int d^{2} z\left[h_{\alpha} \bar{\partial} \theta^{\alpha}+\Omega_{\alpha} \bar{\partial} \Lambda^{\alpha}-\left(\psi^{m}-\frac{1}{2} \Lambda \gamma^{m} \theta\right)\left(\Lambda \gamma_{m}\right)_{\alpha} \bar{\partial} \theta^{\alpha}+\frac{1}{2} \pi^{m} \bar{\pi}_{m}+B_{z \bar{z}}^{\text {het }}\right]
\end{aligned}
$$

where $\Omega_{\alpha}$ and $h_{\alpha}$ are constrained to satisfy $\bar{\lambda} \gamma^{m} \Omega=\bar{\lambda} \gamma^{m} h=0$. Finally, one can define

$$
\Omega_{\alpha}=w_{\alpha}-\frac{1}{2(\lambda \bar{\lambda})}\left(w \gamma_{m} \bar{\lambda}\right)\left(\gamma^{m} \lambda\right), \quad d_{\alpha}=h_{\alpha}-\left(\Lambda \gamma_{m}\right)_{\alpha}\left(\psi^{m}-\frac{1}{2} \Lambda \gamma^{m} \theta\right)
$$

to obtain the heterotic pure spinor action of (2.1)

$$
\begin{aligned}
S & =\int d^{2} z\left[d_{\alpha} \bar{\partial} \theta^{\alpha}+w_{\alpha} \bar{\partial} \lambda^{\alpha}+\frac{1}{2} \pi^{m} \bar{\pi}_{m}+B_{z \bar{z}}^{\mathrm{het}}\right] \\
& =\int d^{2} z\left[\frac{1}{2} \partial x^{m} \bar{\partial} x_{m}+p_{\alpha} \bar{\partial} \theta^{\alpha}+w_{\alpha} \bar{\partial} \lambda^{\alpha}\right]
\end{aligned}
$$

where the relation of $p_{\alpha}$ and $d_{\alpha}$ is defined in (2.3). 
For the Type II superstring, one generalizes the $\mathrm{N}=(1,0)$ superfields of (2.13) to $\mathrm{N}=(1,1)$ off-shell superfields

$$
\begin{aligned}
X^{m} & =x^{m}+\kappa \psi^{m}+\bar{\kappa} \widehat{\psi}^{m}+\kappa \bar{\kappa} f^{m}, & & \\
\Theta^{\alpha} & =\theta^{\alpha}+\kappa \Lambda^{\alpha}+\bar{\kappa} \rho^{\alpha}+\kappa \bar{\kappa} s^{\alpha}, & & \widehat{\Theta}^{\hat{\alpha}}=\widehat{\theta}^{\hat{\alpha}}+\bar{\kappa} \widehat{\Lambda}^{\hat{\alpha}}+\kappa \widehat{\rho}^{\hat{\alpha}}+\kappa \bar{\kappa} \widehat{s}^{\hat{\alpha}}, \\
\Phi_{\alpha} & =\Omega_{\alpha}+\kappa h_{\alpha}+\bar{\kappa} r_{\alpha}+\kappa \bar{\kappa} \xi_{\alpha}, & & \widehat{\Phi}_{\hat{\alpha}}=\widehat{\Omega}_{\hat{\alpha}}+\bar{\kappa} \widehat{h}_{\hat{\alpha}}+\kappa \widehat{r}_{\hat{\alpha}}+\kappa \bar{\kappa} \widehat{\xi}_{\hat{\alpha}},
\end{aligned}
$$

where $(z, \bar{z}, \kappa, \bar{\kappa})$ are the parameters of $\mathrm{N}=(1,1)$ worldsheet superspace and $\alpha$ and $\hat{\alpha}$ denote spinors of the same/opposite chirality for the Type IIB/IIA superstring. In terms of these $\mathrm{N}=(1,1)$ worldsheet superfields, the Type II worldsheet supersymmetric action in a flat background is

$$
\begin{aligned}
S= & \int d^{2} z d \kappa d \bar{\kappa}\left[-\Phi_{\alpha} \bar{D} \Theta^{\alpha}+\widehat{\Phi}_{\hat{\alpha}} D \widehat{\Theta}^{\hat{\alpha}}+\frac{1}{2} \Pi_{\kappa}^{m} \bar{\Pi}_{\bar{\kappa} m}+B_{\kappa \bar{\kappa}}^{I I}\right. \\
& \left.+\left(\bar{\lambda} \gamma_{m} L\right) \Pi_{k}^{m}+M_{m}\left(\bar{\lambda} \gamma^{m} \Phi\right)+\left(\widehat{\bar{\lambda}} \gamma_{m} \widehat{L}\right) \bar{\Pi}_{\bar{\kappa}}^{m}+\widehat{M}_{m}\left(\widehat{\bar{\lambda}} \gamma^{m} \widehat{\Phi}\right)\right]
\end{aligned}
$$

where $\left[L_{\alpha}, M^{m}\right]$ and $\left[\widehat{L}_{\hat{\alpha}}, \widehat{M}^{m}\right]$ are Lagrange multipliers for the left and right-moving constraints

$$
\Pi_{\kappa}^{m}\left(\gamma^{m} \bar{\lambda}\right)^{\alpha}=0, \quad \bar{\lambda} \gamma^{m} \Phi=0, \quad \bar{\Pi}_{\bar{\kappa}}^{m}\left(\gamma^{m} \widehat{\bar{\lambda}}\right)^{\hat{\alpha}}=0, \quad \widehat{\bar{\lambda}} \gamma^{m} \widehat{\Phi}=0,
$$

$\bar{\lambda}_{\alpha}$ and $\widehat{\bar{\lambda}}_{\hat{\alpha}}$ are two fixed pure spinors satisfying $\bar{\lambda}_{\alpha} \lambda^{\alpha} \neq 0$ and $\widehat{\bar{\lambda}}_{\hat{\alpha}} \widehat{\lambda}^{\hat{\alpha}} \neq 0, D=\frac{\partial}{\partial \kappa}+\kappa \frac{\partial}{\partial z}$ and $\bar{D}=\frac{\partial}{\partial \bar{\kappa}}+\bar{\kappa} \frac{\partial}{\partial \bar{z}}$,

$$
\begin{aligned}
\Pi_{\kappa}^{m}= & D X^{m}-\frac{1}{2} D \Theta \gamma^{m} \Theta-\frac{1}{2} D \widehat{\Theta} \gamma^{m} \widehat{\Theta}, \quad \Pi_{z}^{m}=\partial X^{m}-\frac{1}{2} \partial \Theta \gamma^{m} \Theta-\frac{1}{2} \partial \widehat{\Theta} \gamma^{m} \widehat{\Theta} \\
\bar{\Pi}_{\bar{\kappa}}^{m}= & \bar{D} X^{m}-\frac{1}{2} \bar{D} \Theta \gamma^{m} \Theta-\frac{1}{2} \bar{D} \widehat{\Theta} \gamma^{m} \widehat{\Theta}, \quad \bar{\Pi}_{\bar{z}}^{m}=\bar{\partial} X^{m}-\frac{1}{2} \bar{\partial} \Theta \gamma^{m} \Theta-\frac{1}{2} \bar{\partial} \widehat{\Theta} \gamma^{m} \widehat{\Theta} \\
B_{\kappa \bar{\kappa}}^{I I}= & \frac{1}{4}\left[\left(D \Theta \gamma_{m} \Theta-D \widehat{\Theta} \gamma_{m} \widehat{\Theta}\right) \bar{D} X^{m}-D X^{m}\left(\bar{D} \Theta \gamma_{m} \Theta-\bar{D} \widehat{\Theta} \gamma_{m} \widehat{\Theta}\right)\right. \\
& \left.-\frac{1}{2}\left(D \Theta \gamma_{m} \Theta\right)\left(\bar{D} \widehat{\Theta} \gamma^{m} \widehat{\Theta}\right)+\frac{1}{2}\left(D \widehat{\Theta} \gamma^{m} \widehat{\Theta}\right)\left(\bar{D} \Theta \gamma_{m} \Theta\right)\right] \\
B_{z \bar{z}}^{I I}= & \frac{1}{4}\left[\left(\partial \Theta \gamma_{m} \Theta-\partial \widehat{\Theta} \gamma_{m} \widehat{\Theta}\right) \bar{\partial} X^{m}-\partial X^{m}\left(\bar{\partial} \Theta \gamma_{m} \Theta-\bar{\partial} \widehat{\Theta} \gamma_{m} \widehat{\Theta}\right)\right. \\
& \left.-\frac{1}{2}\left(\partial \Theta \gamma_{m} \Theta\right)\left(\bar{\partial} \widehat{\Theta} \gamma^{m} \widehat{\Theta}\right)+\frac{1}{2}\left(\partial \widehat{\Theta} \gamma^{m} \widehat{\Theta}\right)\left(\bar{\partial} \Theta \gamma_{m} \Theta\right)\right]
\end{aligned}
$$

and $B_{\kappa \bar{\kappa}}^{I I}$ is the usual Type II Green-Schwarz two-form $B_{z \bar{z}}$ field with $\frac{\partial}{\partial z}$ and $\frac{\partial}{\partial \bar{z}}$ replaced by $D$ and $\bar{D}$.

After shifting $\Phi_{\alpha}$ and $\widehat{\Phi}_{\hat{\alpha}}$, integrating over $\kappa$ and $\bar{\kappa}$, and solving for auxiliary fields, the action of $(2.22)$ reduces to

$$
\begin{aligned}
S= & \int d^{2} z\left[h_{\alpha} \bar{\partial} \theta^{\alpha}+\Omega_{\alpha} \bar{\partial} \Lambda^{\alpha}+\widehat{h}_{\hat{\alpha}} \partial \widehat{\theta}^{\hat{\alpha}}+\widehat{\Omega}_{\hat{\alpha}} \partial \widehat{\Lambda}^{\hat{\alpha}}-\left(\psi^{m}-\frac{1}{2} \Lambda \gamma^{m} \theta\right)\left(\Lambda \gamma_{m}\right)_{\alpha} \bar{\partial} \theta^{\alpha}\right. \\
& \left.-\left(\widehat{\psi}^{m}-\frac{1}{2} \widehat{\Lambda} \gamma^{m} \widehat{\theta}\right)\left(\widehat{\Lambda} \gamma_{m}\right)_{\alpha} \partial \widehat{\theta}^{\alpha}+\frac{1}{2} \pi^{m} \bar{\pi}_{m}+B_{z \bar{z}}^{I I}\right]
\end{aligned}
$$


where $\left[\Omega_{\alpha}, \widehat{\Omega}_{\hat{\alpha}}, h_{\alpha}, \widehat{h}_{\hat{\alpha}}\right]$ are constrained to satisfy $\bar{\lambda} \gamma^{m} \Omega=\widehat{\bar{\lambda}} \gamma^{m} \widehat{\Omega}=\bar{\lambda} \gamma^{m} h=\widehat{\bar{\lambda}} \gamma^{m} \widehat{h}=0$. Defining

$$
\begin{aligned}
& \Omega_{\alpha}=w_{\alpha}-\frac{1}{2(\lambda \bar{\lambda})}\left(w \gamma_{m} \bar{\lambda}\right)\left(\gamma^{m} \lambda\right), \quad d_{\alpha}=h_{\alpha}-\left(\Lambda \gamma_{m}\right)_{\alpha}\left(\psi^{m}-\frac{1}{2} \Lambda \gamma^{m} \theta\right), \\
& \widehat{\Omega}_{\hat{\alpha}}=\widehat{w}_{\hat{\alpha}}-\frac{1}{2(\widehat{\lambda} \widehat{\bar{\lambda}})}\left(\widehat{w} \gamma_{m} \widehat{\bar{\lambda}}\right)\left(\gamma^{m} \widehat{\lambda}\right), \quad \widehat{d}_{\alpha}=\widehat{h}_{\hat{\alpha}}-\left(\widehat{\Lambda} \gamma_{m}\right)_{\hat{\alpha}}\left(\widehat{\psi}^{m}-\frac{1}{2} \widehat{\Lambda}^{m} \widehat{\theta}\right),
\end{aligned}
$$

one obtains the Type II pure spinor action

$$
\begin{aligned}
S & =\int d^{2} z\left[\frac{1}{2} \pi^{m} \bar{\pi}_{m}+B_{z \bar{z}}^{I I}+d_{\alpha} \bar{\partial} \theta^{\alpha}+w_{\alpha} \bar{\partial} \lambda^{\alpha}+\widehat{d}_{\hat{\alpha}} \partial \widehat{\theta}^{\hat{\alpha}}+\widehat{w}_{\hat{\alpha}} \partial \widehat{\lambda}^{\hat{\alpha}}\right] \\
& =\int d^{2} z\left[\frac{1}{2} \partial x^{m} \bar{\partial} x_{m}+p_{\alpha} \bar{\partial} \theta^{\alpha}+w_{\alpha} \bar{\partial} \lambda^{\alpha}+\widehat{p}_{\hat{\alpha}} \partial \widehat{\theta}^{\hat{\alpha}}+\widehat{w}_{\hat{\alpha}} \partial \widehat{\lambda}^{\hat{\alpha}}\right] .
\end{aligned}
$$

Although the manifestly worldsheet supersymmetric actions of (2.14) and (2.22) are not manifestly Lorentz-covariant because of the presence of $\bar{\lambda}_{\alpha}$ in the constraints of (2.12), one can solve these constraints to obtain the manifestly Lorentz-covariant action of the pure spinor formalism which is, however, not manifestly worldsheet supersymmetric. As will be shown later, there are alternative ways to solve the constraints of (2.12) which either lead to the extended RNS formalism or to the twistor string formalism. However, before discussing the relation of (2.14) and (2.22) to the extended RNS and twistor-string formalisms, it will be shown how to construct vertex operators and compute tree-level scattering amplitudes using this $\mathrm{N}=1$ worldsheet supersymmetric description of the pure spinor formalism.

\section{$2.3 \quad \mathrm{U}(1)$ generator}

As expected for an $\mathrm{N}=1$ superconformal field theory, physical vertex operators $V$ should be $\mathrm{N}=1$ superconformal primary fields of conformal weight $+\frac{1}{2}$ so that the integrated vertex operator $\int G V=\int d z d \kappa V$ is $\mathrm{N}=1$ superconformally invariant. But after imposing the constraints of (2.12) and fixing the $\mathrm{N}=1$ superconformal invariance, the superfields $\left[X^{m}, \Theta^{\alpha}, \Phi_{\alpha}\right]$ contain $30+30$ worldsheet variables. So one needs to impose additional requirements if one wants to reproduce the usual superstring spectrum depending in lightcone gauge on only $8+8$ worldsheet variables.

To obtain the additional requirements, consider the $\mathrm{U}(1)$ generator

$$
J=-\lambda^{\alpha} w_{\alpha}
$$

which has the OPE's

$$
J(y) G(z) \rightarrow(y-z)^{-1}\left(G^{+}-G^{-}\right)
$$

where $G=G^{+}+G^{-}$and $G^{ \pm}$are defined in (2.2) and carry $\pm 1 \mathrm{U}(1)$ charge with respect to $\oint J$. Since the integrated vertex operator $\int G V$ is $\mathrm{N}=1$ superconformally invariant, it would be $\mathrm{N}=2$ superconformally invariant if it had no poles with $J$ since this would imply that $\int G V$ has no poles with either $G^{+}$or $G^{-}$. Although this condition on the vertex operator would be too restrictive, an appropriate condition is that $\int G V$ must have only 
terms of zero or negative $\mathrm{U}(1)$ charge with respect to $\oint J$. Defining $\int(G V)_{n}$ to be the term in $\int G V$ with $\mathrm{U}(1)$ charge $n$, this condition combined with $\mathrm{N}=1$ superconformal invariance implies that $\left[\oint G^{+}, \int(G V)_{0}\right]=0$.

It will later be shown that charge conservation implies that the terms with negative $\mathrm{U}(1)$ charge in $\int G V$ do not contribute to tree amplitudes. So at least for tree amplitudes, the integrated vertex operator can be identified with $\int(G V)_{0}$ which is annihilated by $\oint G^{+}$. Furthermore, it will be required that the integrated vertex operator of zero U(1) charge, $\int(G V)_{0}$, is independent of the fixed pure spinor $\bar{\lambda}_{\alpha}$ and is therefore globally defined on the pure spinor space. So in addition to requring that $\int G V$ is $\mathrm{N}=1$ superconformally invariant, it will also be required that $\int(G V)_{n}=0$ for $n$ positive and that $\int(G V)_{0}$ is globally defined on the pure spinor space, i.e. $\int(G V)_{0}$ is independent of $\bar{\lambda}_{\alpha}$ and is invariant under the gauge transformation $\delta w_{\alpha}=f^{m}\left(\gamma_{m} \lambda\right)_{\alpha}$. By fixing the way that the vertex operator depends on 11 components of $\lambda^{\alpha}$ and $\theta^{\alpha}$ and their conjugate momenta, these additional requirements will reduce the degrees of freedom in physical vertex operators from $30+30$ worldsheet variables to $8+8$ worldsheet variables.

\subsection{Massless vertex operators}

$\mathrm{N}=1$ superconformal invariance implies that the open superstring unintegrated massless vertex operator of conformal weight $+\frac{1}{2}$ has the form

$$
V=D \Theta^{\alpha} A_{\alpha}(X, \Theta)+\Pi_{\kappa}^{m} A_{m}(X, \Theta)+\Phi_{\alpha} W^{\alpha}(X, \Theta)
$$

where $\left(A_{\alpha}, A_{m}, W^{\alpha}\right)$ are spacetime superfields with momentum $k^{m}$ satisfying $k^{m} k_{m}=0$. By acting on $V$ with the worldsheet superspace derivative $D$, the integrated vertex operator is easily computed to be

$$
\begin{aligned}
G V= & \partial \Theta^{\alpha} A_{\alpha}+\Pi_{z}^{m} A_{m}+D \Phi_{\alpha} W^{\alpha}+\Phi_{\alpha} D \Theta^{\beta} \nabla_{\beta} W^{\alpha}+\Phi_{\alpha} \Pi_{\kappa}^{m} \partial_{m} W^{\alpha} \\
& +D \Theta^{\alpha} D \Theta^{\beta}\left(-\frac{1}{2} A_{m} \gamma_{\alpha \beta}^{m}+\nabla_{\beta} A_{\alpha}\right)+D \Theta^{\alpha} \Pi_{\kappa}^{m}\left(\partial_{m} A_{\alpha}-\nabla_{\alpha} A_{m}\right)+\Pi_{\kappa}^{m} \Pi_{\kappa}^{n} \partial_{m} A_{n} .
\end{aligned}
$$

The constraints of (2.12) imply that the $\kappa=0$ component of the superfields $\Phi_{\alpha}$ and $\Pi_{\kappa}^{m}$ carry $-1 \mathrm{U}(1)$ charge, and the condition that $(G V)_{2}=0$ implies the $\mathrm{d}=10$ super-Yang-Mills equation of motion $\gamma_{m_{1} \ldots m_{5}}^{\alpha \beta} \nabla_{\alpha} A_{\beta}=0$. So

$$
\begin{aligned}
(G V)_{0}= & \partial \theta^{\alpha} A_{\alpha}+\pi^{m} A_{m}+h_{\alpha} W^{\alpha}+\lambda^{\beta} \Omega_{\alpha} \nabla_{\beta} W^{\alpha} \\
& +\lambda^{\alpha} \pi_{n} \frac{\left(\gamma^{n} \bar{\lambda}\right)^{\beta}}{\lambda \bar{\lambda}}\left(-A_{m} \gamma_{\alpha \beta}^{m}+\nabla_{\beta} A_{\alpha}+\nabla_{\alpha} A_{\beta}\right)+\lambda^{\alpha} \Pi_{\kappa}^{m}\left(\partial_{m} A_{\alpha}-\nabla_{\alpha} A_{m}\right) .
\end{aligned}
$$

Using the definitions of (2.19), one can verify that $(G V)_{0}$ is independent of $\bar{\lambda}_{\alpha}$ if

$$
\nabla_{\alpha} A_{\beta}+\nabla_{\beta} A_{\alpha}=\gamma_{\alpha \beta}^{m} A_{m}, \quad \nabla_{\alpha} A_{m}-\partial_{m} A_{\alpha}=\gamma_{m \alpha \beta} W^{\beta},
$$

which are the usual onshell superfield constraints for $d=10$ super-Yang-Mills. And after imposing these super-Yang-Mills constraints, $(G V)_{0}$ reproduces the pure spinor integrated vertex operator

$$
U=(G V)_{0}=\partial \theta^{\alpha} A_{\alpha}+\pi^{m} A_{m}+d_{\alpha} W^{\alpha}+\frac{1}{4}\left(w \gamma^{m n} \lambda\right) F_{m n}
$$


where $\nabla_{\alpha} W^{\beta}=\frac{1}{4}\left(\gamma^{m n}\right)_{\alpha}^{\beta} F_{m n}$ and $d_{\alpha} \equiv h_{\alpha}-\left(\lambda \gamma_{m}\right)_{\alpha} \Pi_{\kappa}^{m}$ differs from the definition of $d_{\alpha}$ in (2.19) by a term with $-2 \mathrm{U}(1)$ charge which does not contribute to (2.37). Note that $(G V)_{-2}$ is nonzero and satisfies $G^{-} \int(G V)_{0}=-G^{+} \int(G V)_{-2}$. This explains why the usual pure spinor integrated vertex operator $U$ of (2.37) is not annihilated by the $b$ ghost [20] but satisfies $b_{-1} \int U=Q \int \Lambda$ where $\Lambda=-(G V)_{-2} \cdot{ }^{1}$

\subsection{Tree-level scattering amplitudes}

In the pure spinor formalism, the usual tree-level $N$-point open string scattering amplitude prescription is to take 3 vertex operators $V_{r}$ of ghost-number one and conformal weight zero, and $N-3$ integrated vertex operator $U_{r}$ of ghost-number zero and conformal weight one. One then defines the tree amplitude $A$ to be the correlation function

$$
A=\left\langle V_{1}\left(z_{1}\right) V_{2}\left(z_{2}\right) V_{3}\left(z_{3}\right) \int d z_{4} U_{4} \ldots \int d z_{N} U_{N}\right\rangle
$$

where the $\left(z_{1}, z_{2}, z_{3}\right)$ are arbitrary points and the zero mode normalization is defined by $\left\langle\left(\lambda \gamma^{m} \theta\right)\left(\lambda \gamma^{n} \theta\right)\left(\lambda \gamma^{p} \theta\right)\left(\theta \gamma_{m n p} \theta\right)\right\rangle=1$. Although this prescription only requires 5 of the 16 $\theta$ zero modes to be present in the integrand, it is spacetime supersymmetric since one can show that any term in the integrand with more than $5 \theta$ zero modes and +3 ghost-number is not in the cohomology of $Q=\int \lambda^{\alpha} d_{\alpha}[1]$.

But before twisting, the vertex operators $V_{r}$ of +1 ghost-number have conformal weight $+\frac{1}{2}$. So this prescription is only conformally invariant after twisting the pure spinor formalism and is inconsistent in the untwisted pure spinor formalism. Fortunately, there is an alternative prescription one can define for tree-level amplitudes in the pure spinor formalism which only involves ghost-number zero vertex operators $U$ and can be defined both before and after twisting.

In this alternative prescription, one takes $N$ integrated vertex operators $U_{r}$ of ghostnumber zero and conformal weight one and defines the tree amplitude as

$$
A=\left\langle\left(z_{1}-z_{2}\right)\left(z_{2}-z_{3}\right)\left(z_{3}-z_{1}\right) U_{1}\left(z_{1}\right) U_{2}\left(z_{2}\right) U_{3}\left(z_{3}\right) \int d z_{4} U_{4} \ldots \int d z_{N} U_{N}\right\rangle
$$

where $\left(z_{1}, z_{2}, z_{3}\right)$ are arbitrary points and the zero mode normalization is defined by $\langle 1\rangle=1$. In this prescription, none of the $16 \theta$ zero modes need to be present in the integrand. But it is again spacetime supersymmetric since one can show that the only term in the cohomology of $Q=\int \lambda^{\alpha} d_{\alpha}$ with zero ghost-number is the identity operator. So any term with $\theta$ zero modes and zero ghost number will decouple since it is not in the cohomology of $Q=\int \lambda^{\alpha} d_{\alpha}$.

For example, consider the $N$-point Yang-Mills tree amplitude where $U$ is defined in (2.37). For $N$ external gluons, requiring an equal number of $\theta^{\alpha}$ and $p_{\alpha}$ zero modes implies that the only term in (2.37) which contributes is

$$
U=\partial x^{m} A_{m}(x)+\frac{1}{2} M^{m n} F_{m n}(x)
$$

\footnotetext{
${ }^{1}$ An interesting question is which vertex operators satisfy $(G V)_{-2}=0$ and therefore are annihilated by the $b$ ghost and preserve $\mathrm{N}=2$ worldsheet supersymmetry. By analyzing (2.34), one finds that $(G V)_{-2}=0$ if and only if $\bar{\lambda}_{\alpha} W^{\alpha}=0$. Since $\nabla_{\beta} W^{\alpha}=\frac{1}{4}\left(\gamma^{m n}\right)^{\alpha}{ }_{\beta} F_{m n}, \bar{\lambda}_{\alpha} W^{\alpha}=0$ implies that $F_{m n}\left(\gamma^{m n} \bar{\lambda}\right)_{\alpha}=0$, so $\bar{\lambda}_{\alpha}$ is a killing spinor in these backgrounds. I would like to thank Andrei Mikhailov for discussions on this point.
} 
where $M^{m n}=\frac{1}{2}\left(p \gamma^{m n} \theta\right)+\frac{1}{2}\left(w \gamma^{m n} \lambda\right)$. Using the fact that $M^{m n}$ is a Lorentz current of level 1 with the same OPE's as the RNS Lorentz current $\psi^{m} \psi^{n}$, one can easily verify that the prescription of (2.39) reproduces the correct tree amplitudes.

A similar zero mode prescription was used by Lee and Siegel in [21], and is closely related to the $\mathcal{F}_{1}$ picture for scattering Neveu-Schwarz states in the RNS formalism. To compute $N$-point open string RNS tree amplitudes in this $\mathcal{F}_{1}$ picture, one chooses all $N$ Neveu-Schwarz vertex operators in the zero picture and uses the same zero mode regularization $\left\langle c\left(z_{1}\right) c\left(z_{2}\right) c\left(z_{3}\right)\right\rangle=\left(z_{1}-z_{2}\right)\left(z_{2}-z_{3}\right)\left(z_{3}-z_{1}\right)$ as in the bosonic string. Although it is unclear how to generalize this prescription to loop amplitudes in the RNS formalism, it is easy to show that computations in the $\mathcal{F}_{1}$ picture reproduce the same tree-level amplitude prescription as in the conventional $\mathcal{F}_{2}$ picture [22] where two Neveu-Schwarz vertex operators are chosen in the -1 picture and one uses the zero mode regularization

$$
\left\langle c\left(z_{1}\right) e^{-\phi\left(z_{1}\right)} c\left(z_{2}\right) e^{-\phi\left(z_{2}\right)} c\left(z_{3}\right)\right\rangle=\left(z_{1}-z_{3}\right)\left(z_{2}-z_{3}\right) .
$$

To prove the equivalence of RNS computations in the $\mathcal{F}_{1}$ and $\mathcal{F}_{2}$ pictures, multiply the BRST-invariant state $\left(c \partial c \partial^{2} c e^{-2 \phi}\right)$ appearing in (2.41) with two picture raising operators to obtain a BRST-invariant state of ghost-number three and zero picture which includes the term $c \partial c \partial^{2} c$.

So for computing tree-level scattering amplitudes in the untwisted pure spinor formalism, the prescription of (2.39) can be used. Because of $U(1)$ charge conservation with respect to $J=-\lambda^{\alpha} w_{\alpha}$ and the absence of terms with positive $\mathrm{U}(1)$ charge in the worldsheet action and vertex operators, terms with negative $\mathrm{U}(1)$ charge cannot contribute to tree amplitudes using this precription. Furthermore, it will be verified in the next section that the tree amplitude prescription of (2.39) for Neveu-Schwarz states in the extended RNS formalism gives the same tree amplitudes as in the usual RNS formalism. Although it will not be verified here, it is natural to conjecture that the prescription of (2.39) with the zero mode normalization $\langle 1\rangle=1$ can also be used to compute twistor-string tree amplitudes.

\subsection{Extended RNS formalism}

The $\mathrm{N}=1$ worldsheet superfields in the pure spinor formalism are $\left[X^{m}, \Theta^{\alpha}, \Phi_{\alpha}\right]$ satisfying the constraints of $(2.12)$ that $\left(\gamma^{m} \bar{\lambda}\right)^{\alpha}\left(D X_{m}-\frac{1}{2} D \Theta \gamma_{m} \Theta\right)=0$ and $\left(\bar{\lambda} \gamma^{m} \Phi\right)=0$. If one shifts $\Theta^{\alpha}$ by defining

$$
\Theta^{\prime \alpha} \equiv \Theta^{\alpha}+K_{m}\left(\gamma^{m} \bar{\lambda}\right)^{\alpha} \text { where } K^{m}=-\frac{1}{\bar{\lambda} D \Theta^{\prime}} D X^{m}+\frac{1}{2} \frac{\bar{\lambda} \Theta^{\prime}}{\bar{\lambda} D \Theta^{\prime}} D\left(\frac{D X^{m}}{\bar{\lambda} D \Theta^{\prime}}\right),
$$

$\left(\gamma^{m} \bar{\lambda}\right)^{\alpha}\left(D X_{m}-\frac{1}{2} D \Theta \gamma_{m} \Theta\right)=0$ implies that $\left(\bar{\lambda} \gamma_{m}\right)^{\alpha}\left(\Theta^{\prime} \gamma^{m} D \Theta^{\prime}\right)=0$. So the $\mathrm{N}=1$ superfields $\left[\Theta^{\prime \alpha}, \Phi_{\alpha}\right]$ satisfy the constraints

$$
\left(\bar{\lambda} \gamma_{m}\right)^{\alpha}\left(\Theta^{\prime} \gamma^{m} D \Theta^{\prime}\right)=0, \quad \bar{\lambda} \gamma^{m} \Phi=0
$$

and leave unconstrained the $\mathrm{N}=1$ superfield $X^{m}$. Interpreting $X^{m}$ as the usual $\mathrm{N}=1$ worldsheet superfield of the RNS formalism, the formalism including both $X^{m}$ and $\left(\Theta^{\prime \alpha}, \Phi_{\alpha}\right)$ will 
be called the "extended RNS formalism". In components, it is convenient to expand the new superfield $\Theta^{\prime \alpha}$ as

$$
\Theta^{\prime \alpha}=\theta^{\prime \alpha}+\kappa \lambda^{\alpha}+\left(\gamma^{m} \bar{\lambda}\right)^{\alpha}\left(f_{m}+\kappa g_{m}\right)
$$

where $\theta^{\prime \alpha}$ and $\lambda^{\alpha}$ are constrained to satisfy

$$
\theta^{\prime} \gamma^{m} \lambda=0, \quad \lambda \gamma^{m} \lambda=0
$$

and $\left(\bar{\lambda} \gamma_{m}\right)^{\alpha}\left(\Theta^{\prime} \gamma^{m} D \Theta^{\prime}\right)=0$ implies that $f_{m}$ and $g_{m}$ are quadratic and higher-order in $\theta^{\prime \alpha}$.

In terms of the $\mathrm{N}=(1,0)$ superfields $\left[X^{m}, \Theta^{\prime \alpha}, \Phi_{\alpha}\right]$, the heterotic worldsheet action in the extended RNS formalism will be defined to be the sum of the usual RNS action with an action for the $\left[\Theta^{\prime \alpha}, \Phi_{\alpha}\right]$ superfields as

$$
S=\int d^{2} z d \kappa\left[\frac{1}{2} D X^{m} \bar{\partial} X_{m}+\Phi_{\alpha} \bar{\partial} \Theta^{\prime \alpha}+\left(\bar{\lambda} \gamma_{m} L\right)\left(\Theta^{\prime} \gamma^{m} D \Theta^{\prime}\right)+M_{m}\left(\bar{\lambda} \gamma^{m} \Phi\right)\right] .
$$

And in terms of the $\mathrm{N}=(1,1)$ superfields $\left[X^{m}, \Theta^{\prime \alpha}, \widehat{\Theta}^{\prime \hat{\alpha}}, \Phi_{\alpha}, \widehat{\Phi}_{\hat{\alpha}}\right]$, the Type II worldsheet action will be defined as

$$
\begin{aligned}
S= & \int d^{2} z d \kappa d \bar{\kappa}\left[\frac{1}{2} D X^{m} \bar{D} X_{m}-\Phi_{\alpha} \bar{D} \Theta^{\prime \alpha}+\widehat{\Phi}_{\hat{\alpha}} D \widehat{\Theta}^{\prime \hat{\alpha}}\right. \\
& \left.+\left(\bar{\lambda} \gamma_{m} L\right)\left(\Theta^{\prime} \gamma^{m} D \Theta^{\prime}\right)+M_{m}\left(\bar{\lambda} \gamma^{m} \Phi\right)+\left(\widehat{\bar{\lambda}} \gamma_{m} \widehat{L}\right)\left(\widehat{\Theta}^{\prime} \gamma^{m} \bar{D} \widehat{\Theta}^{\prime}\right)+\widehat{M}_{m}\left(\widehat{\bar{\lambda}} \gamma^{m} \widehat{\Phi}\right)\right] .
\end{aligned}
$$

To relate the pure spinor heterotic action of (2.14) to the extended RNS heterotic action of (2.46), substitute into (2.18) the component form of (2.42) which is

$$
\theta^{\alpha}=\theta^{\alpha}-\left[\frac{1}{\bar{\lambda} \lambda} \psi^{m}-\frac{\left(\bar{\lambda} \theta^{\prime}\right)}{2(\bar{\lambda} \lambda)^{2}}\left(\partial x^{m}-\psi^{m} \frac{\left(\bar{\lambda} \partial \theta^{\prime}\right)}{(\bar{\lambda} \lambda)}\right)+f^{m}\right]\left(\gamma_{m} \bar{\lambda}\right)^{\alpha}
$$

where $f_{m}$ is quadratic and higher-order in $\theta^{\prime \alpha}$. In terms of $\theta^{\prime \alpha}$, the action of $(2.18)$ is

$$
\begin{aligned}
S & =\int d^{2} z\left[h_{\alpha} \bar{\partial} \theta^{\prime \alpha}+\Omega_{\alpha} \bar{\partial} \lambda^{\alpha}-\psi_{m}\left(\lambda \gamma^{m} \gamma^{n} \bar{\lambda}\right) \bar{\partial}\left(\frac{1}{\lambda \bar{\lambda}} \psi^{n}-\frac{\left(\bar{\lambda} \theta^{\prime}\right)}{2(\bar{\lambda} \lambda)^{2}} \partial x^{n}\right)+\frac{1}{2} \partial x^{m} \bar{\partial} x_{m}+O\left(\theta^{\prime}\right)\right] \\
& =\int d^{2} z\left[\left(h_{\alpha}-\bar{\lambda}_{\alpha} r^{m} \partial x_{m}\right) \bar{\partial} \theta^{\prime \alpha}+\left(\Omega_{\alpha}-2 \psi_{m} r^{m} \bar{\lambda}_{\alpha}\right) \bar{\partial} \lambda^{\alpha}-\psi_{m} \bar{\partial} \psi^{m}+\frac{1}{2} \partial x^{m} \bar{\partial} x_{m}+O\left(\theta^{\prime}\right)\right]
\end{aligned}
$$

where $O\left(\theta^{\prime}\right)$ denotes terms linear or higher-order in $\theta^{\prime \alpha}$ (counting its conjugate momentum $h_{\alpha}$ as an inverse power of $\left.\theta^{\prime \alpha}\right)$ and

$$
r^{m}=\psi_{n} \frac{\bar{\lambda} \gamma^{m} \gamma^{n} \lambda}{2(\lambda \bar{\lambda})^{2}}
$$

Defining $\Phi_{\alpha}=\tilde{\Omega}_{\alpha}+\kappa \tilde{h}_{\alpha}$ in the extended RNS action of (2.46) where

$$
\tilde{\Omega}_{\alpha} \equiv \Omega_{\alpha}-2 \psi_{m} r^{m} \bar{\lambda}_{\alpha}, \quad \tilde{h}_{\alpha} \equiv h_{\alpha}-r^{m} \partial x_{m} \bar{\lambda}_{\alpha},
$$

one can easily verify that (2.46) reproduces (2.49) if one ignores terms proportional to $O\left(\theta^{\prime}\right)$. 
To understand why these $O\left(\theta^{\prime}\right)$ terms can be ignored, note that physical vertex operators will be required to carry zero $\mathrm{U}(1)$ charge with respect to $J=-\lambda^{\alpha} \tilde{\Omega}_{\alpha}$ and be globally defined on the pure spinor space, i.e. physical vertex operators must be independent of $\bar{\lambda}_{\alpha}$ and be invariant under the gauge transformations

$$
\delta \tilde{\Omega}_{\alpha}=\xi_{m}\left(\gamma^{m} \lambda\right)_{\alpha}+\rho_{m}\left(\gamma^{m} \theta^{\prime}\right)_{\alpha}, \quad \delta \tilde{h}_{\alpha}=\left(\gamma^{m} \lambda\right)_{\alpha} \rho_{m},
$$

generated by the constraints of $(2.45)$ where $\xi_{m}$ and $\rho_{m}$ are arbitrary parameters. It can be verified that all quantities with zero $\mathrm{U}(1)$ charge which are gauge-invariant under (2.52) must contain non-negative powers of $\theta^{\prime \alpha}$ (where $\tilde{h}_{\alpha}$ counts as an inverse power of $\theta^{\prime \alpha}$ ), e.g. the Lorentz current $M^{m n}=\frac{1}{2}\left(\tilde{\Omega} \gamma^{m n} \lambda+\tilde{h} \gamma^{m n} \theta^{\prime}\right)$. And since the tree amplitude prescription vanishes unless there are an equal number of $\theta^{\prime \alpha}$ 's and $\tilde{h}_{\alpha}$ 's in the correlation function, one can ignore any $O\left(\theta^{\prime}\right)$ terms in the worldsheet action which are linear or higher-order in $\theta^{\prime \alpha}$.

Finally, it will be argued that the computation of tree-level scattering amplitudes of physical states using the prescription of (2.39) in the extended RNS formalism is equivalent to the computation of Neveu-Schwarz states using the usual RNS prescription in the $\mathcal{F}_{1}$ picture. To prove this equivalence, one needs to show that the extra fields $\left(\lambda^{\alpha}, \theta^{\prime \alpha}, \tilde{\Omega}_{\alpha}, \tilde{h}_{\alpha}\right)$ in the extended RNS formalism do not contribute to tree-level scattering amplitudes using the zero mode normalization where $\langle 1\rangle=1$.

Any physical vertex operator in the extended RNS formalism must be gauge-invariant under (2.52) and be a worldsheet primary field with zero U(1) charge. Examples of such gauge-invariant operators with zero U(1) charge which involve $\tilde{h}_{\alpha}$ and $\tilde{\Omega}_{\alpha}$ are $M^{m n}=$ $\frac{1}{2}\left(\tilde{\Omega} \gamma^{m n} \lambda+\tilde{h} \gamma^{m n} \theta^{\prime}\right)$ and its derivatives. But since $M^{m n}$ has level zero, i.e. the OPE of $M^{m n}$ with $M^{p q}$ has no double pole proportional to the identity operator, it is not possible for a correlation function involving $M^{m n}$ and its derivatives to be proportional to the identity operator. It seems reasonable to conjecture that all gauge-invariant opeartors depending on $\tilde{h}_{\alpha}$ or $\tilde{\Omega}_{\alpha}$ are of this type and cannot produce the identity operator in their OPE's. Therefore, any terms in the vertex operator which depend on $\tilde{h}_{\alpha}$ or $\tilde{\Omega}_{\alpha}$ will decouple from the tree amplitudes. Furthermore, since the tree amplitude vanishes unless there are an equal number of $\tilde{h}_{\alpha}$ 's and $\theta^{\prime \alpha}$ 's in the correlation function, any terms in the vertex operator which depend on $\theta^{\prime \alpha}$ will also decouple. So the only terms in the vertex operator which can contribute to tree amplitudes are terms that only depend on the superfield $X^{m}$. But worldsheet $\mathrm{N}=1$ superconformal primary fields which only depend on $X^{m}$ are the usual Neveu-Schwarz states in the RNS formalism. So tree amplitudes of physical states in the extended RNS formalism are equivalent to the tree amplitudes of Neveu-Schwarz states in the usual RNS formalism.

\subsection{Worldsheet supersymmetric action in curved background}

By adding integrated vertex operators to the worldsheet action in a flat target-space background, one can generalize the $\mathrm{N}=1$ worldsheet supersymmetric actions to a curved background. For the heterotic superstring in the pure spinor description, the $\mathrm{N}=(1,0)$ worldsheet supersymmetric action of (2.14) generalizes in an $\mathrm{N}=1 \mathrm{~d}=10$ supergravity 
background to

$$
\begin{aligned}
S= & \int d^{2} z d \kappa\left[\frac{1}{2} \eta_{a b} E_{M}^{a} D Z^{M} E_{N}^{b} \bar{\partial} Z^{N}+\frac{1}{2} B_{M N}^{\text {het }} D Z^{M} \bar{\partial} Z^{N}+\Phi_{\alpha} E_{M}^{\alpha} \bar{\partial} Z^{M}\right. \\
& \left.+\left(\bar{\lambda} \gamma_{a} L\right) E_{M}^{a} D Z^{M}+M_{a}\left(\bar{\lambda} \gamma^{a} \Phi\right)\right]
\end{aligned}
$$

where $M=(m, \mu)$ are curved-space indices for $m=0$ to 9 and $\mu=1$ to $16, A=(a, \alpha)$ are tangent-space indices for $a=0$ to 9 and $\alpha=1$ to $16, Z^{M}=\left(X^{m}, \Theta^{\mu}\right), E_{M}^{A}$ is the super-vierbein, and $B_{M N}^{\text {het }}$ is the graded antisymmetric tensor superfield.

And for the Type II superstring in the pure spinor description, the $\mathrm{N}=(1,1)$ worldsheet supersymmetric action of (2.22) generalizes in an $\mathrm{N}=2 \mathrm{~d}=10$ supergravity background to

$$
\begin{aligned}
S= & \int d^{2} z d \kappa d \bar{\kappa}\left[\frac{1}{2} \eta_{a b} E_{M}^{a} D Z^{M} E_{N}^{b} \bar{D} Z^{N}+\frac{1}{2} B_{M N}^{I I} D Z^{M} \bar{D} Z^{N}\right. \\
& -\Phi_{\alpha} E_{M}^{\alpha} \bar{D} Z^{M}+\widehat{\Phi}_{\hat{\alpha}} E_{M}^{\hat{\alpha}} D Z^{M}-F^{\alpha \hat{\alpha}} \Phi_{\alpha} \widehat{\Phi}_{\hat{\alpha}} \\
& \left.+\left(\bar{\lambda} \gamma_{a} L\right) E_{M}^{a} D Z^{M}+M_{a}\left(\bar{\lambda} \gamma^{a} \Phi\right)+\left(\widehat{\bar{\lambda}} \gamma_{a} \widehat{L}\right) E_{M}^{a} \bar{D} Z^{M}+\widehat{M}_{a}\left(\widehat{\bar{\lambda}} \gamma^{a} \widehat{\Phi}\right)\right]
\end{aligned}
$$

where $M=(m, \mu, \hat{\mu})$ are curved-space indices, $A=(a, \alpha, \hat{\alpha})$ are tangent-space indices, $Z^{M}=\left(X^{m}, \Theta^{\mu}, \widehat{\Theta}^{\hat{\mu}}\right), E_{M}^{A}$ is the super-vierbein, $B_{M N}^{I I}$ is the graded antisymmetric tensor superfield, and $F^{\alpha \hat{\alpha}}$ is the superfield whose lowest components are the Type II RamondRamond bispinor field strengths.

After imposing the constraints from varying the Lagrange multipliers, one can expand the actions of (2.53) and (2.54) in components in terms of the pure spinor worldsheet variables $\left(Z^{M}, d_{\alpha}, \lambda^{\alpha}, w_{\alpha}, \bar{\lambda}_{\alpha}\right)$. Although it will not be verified here, it is expected that when the supergravity fields are onshell, all terms in the action will have either zero or negative $\mathrm{U}(1)$ charge with respect to $(2.31)$, and the terms with zero $\mathrm{U}(1)$ charge will be independent of $\bar{\lambda}_{\alpha}$ and reproduce the pure spinor worldsheet action in a curved background of [23].

Using the extended RNS description, the heterotic superstring action of (2.46) can be generalized in a Neveu-Schwarz background and the resulting action is

$$
\begin{aligned}
S= & \int d^{2} z d \kappa\left[\frac{1}{2}\left(g_{m n}(X)+b_{m n}(X)\right) D X^{m} \bar{\partial} X_{m}+\Phi_{\alpha}\left(\overline{\nabla_{\bar{z}}} \Theta^{\prime}\right)^{\alpha}\right. \\
& \left.+\left(\bar{\lambda} \gamma_{a} L\right)\left(\Theta^{\prime} \gamma^{a} \nabla_{\kappa} \Theta^{\prime}\right)+M_{a}\left(\bar{\lambda} \gamma^{a} \Phi\right)\right]
\end{aligned}
$$

where $\left(\bar{\nabla}_{\bar{z}} \Theta^{\prime}\right)^{\alpha}=\bar{\partial} \Theta^{\prime \alpha}+\bar{\partial} X^{m} \omega_{m \beta}{ }^{\alpha}(X) \Theta^{\prime \beta},\left(\nabla_{\kappa} \Theta^{\prime}\right)^{\alpha}=D \Theta^{\prime \alpha}+D X^{m} \omega_{m \beta}{ }^{\alpha}(X) \Theta^{\prime \beta}$, and $\omega_{n \beta^{\alpha}}$ is the spin connection. Similarly, the Type II worldsheet action of (2.47) generalizes in a Neveu-Schwarz/Neveu-Schwarz background to

$$
\begin{aligned}
S= & \int d^{2} z d \kappa d \bar{\kappa}\left[\frac{1}{2}\left(g_{m n}(X)+b_{m n}(X)\right) D X^{m} \bar{D} X^{n}-\Phi_{\alpha}\left(\bar{\nabla}_{\bar{\kappa}} \Theta^{\prime}\right)^{\alpha}+\widehat{\Phi}_{\hat{\alpha}}\left(\nabla_{\kappa} \widehat{\Theta}^{\prime}\right)^{\hat{\alpha}}\right. \\
& \left.+\left(\bar{\lambda} \gamma_{a} L\right)\left(\Theta^{\prime} \gamma^{a} \nabla_{\kappa} \Theta^{\prime}\right)+M_{a}\left(\bar{\lambda} \gamma^{a} \Phi\right)+\left(\widehat{\bar{\lambda}} \gamma_{a} \widehat{L}\right)\left(\widehat{\Theta}^{\prime} \gamma^{a} \bar{\nabla}_{\bar{\kappa}} \widehat{\Theta}^{\prime}\right)+\widehat{M}_{a}\left(\widehat{\bar{\lambda}} \gamma^{m} \widehat{\Phi}\right)\right],
\end{aligned}
$$

where $\left(\bar{\nabla}_{\bar{\kappa}} \Theta^{\prime}\right)^{\alpha}=\bar{D} \Theta^{\prime \alpha}+\bar{D} X^{m} \omega_{m \beta}{ }^{\alpha}(X) \Theta^{\prime \beta},\left(\nabla_{\kappa} \widehat{\Theta}^{\prime}\right)^{\hat{\alpha}}=D \widehat{\Theta}^{\prime \hat{\alpha}}+D X^{m} \widehat{\omega}_{m \hat{\beta}}{ }^{\hat{\alpha}}(X) \widehat{\Theta}^{\prime \hat{\beta}}$, and $\omega_{n \beta}{ }^{\alpha}$ and $\widehat{\omega}_{n \hat{\beta}}{ }^{\hat{\alpha}}$ are the left and right-moving spin connections. 


\section{Twistor string formalism}

\subsection{Twistor superfields}

By choosing different solutions of the superfield constraints of (2.12),

$$
\left(\gamma^{m} \bar{\lambda}\right)^{\alpha}\left(D X_{m}-\frac{1}{2} D \Theta \gamma_{m} \Theta\right)=0, \quad\left(\bar{\lambda} \gamma^{m}\right)^{\alpha} \Phi_{\alpha}=0,
$$

one obtains different worldsheet supersymmetric descriptions of the superstring. Expanding the superfields in component fields as

$$
X^{m}=x^{m}+\kappa \psi^{m}, \quad \Theta^{\alpha}=\theta^{\alpha}+\kappa \Lambda^{\alpha}, \quad \Phi_{\alpha}=\Omega_{\alpha}+\kappa h_{\alpha},
$$

the pure spinor description solves for $\psi^{m}$ and $h_{\alpha}$ in terms of $d_{\alpha}$ through the equations (2.9) and (2.19). And in the extended RNS description, one solves for $\theta^{\alpha}$ in terms of $\psi^{m}$ and a constrained $\theta^{\prime \alpha}$ satisfying $\left(\lambda \gamma^{m} \theta^{\prime}\right)=0$ by shifting $\Theta^{\alpha}$ to $\Theta^{\prime \alpha}$ as in (2.42). In both of these descriptions, the bosonic component fields $\Lambda^{\alpha}$ and $\Omega_{\alpha}$ are solved in terms of $x^{m}$ and $\left(\lambda^{\alpha}, w_{\alpha}\right)$ where $\lambda^{\alpha}$ is a pure spinor and

$$
\Lambda^{\alpha}=\lambda^{\alpha}+\frac{1}{2(\lambda \bar{\lambda})}\left(\partial x^{m}-\frac{1}{2} \partial \theta \gamma^{m} \theta\right)\left(\gamma_{m} \bar{\lambda}\right)^{\alpha}, \quad \Omega_{\alpha}=w_{\alpha}-\frac{1}{2(\lambda \bar{\lambda})}\left(\bar{\lambda} \gamma^{m} w\right)\left(\gamma_{m} \lambda\right)_{\alpha} .
$$

In this section, a new twistor-like solution for the constraints of (3.1) will be presented in which the superfield $X^{m}=x^{m}+\kappa \psi^{m}$ is solved in terms of the other superfields. In this description, the superfield $\Phi_{\alpha}$ is shifted to $\Phi_{\alpha}^{\prime}=\Phi_{\alpha}-\frac{1}{2} X^{m}\left(\gamma_{m} D \Theta\right)_{\alpha}$ whose components $\Phi_{\alpha}^{\prime} \equiv \Omega_{\alpha}^{\prime}+\kappa h_{\alpha}^{\prime}$ no longer satisfy $\bar{\lambda} \gamma^{m} \Omega^{\prime}=\bar{\lambda} \gamma^{m} h^{\prime}=0$. It will be convenient to expand the bosonic component fields $\Lambda^{\alpha}$ and $\Omega_{\alpha}^{\prime}$ which appear in $\Theta^{\alpha}$ and $\Phi_{\alpha}^{\prime}$ as

$$
\Lambda^{\alpha}=\lambda^{\alpha}+\frac{1}{2(\lambda \bar{\lambda})}\left(\gamma^{m} \bar{\lambda}\right)^{\alpha}\left(\lambda \gamma_{m} \nu\right), \quad \Omega_{\alpha}^{\prime}=\mu_{\alpha}+w_{\alpha}-\frac{1}{2(\lambda \bar{\lambda})}\left(\gamma^{m} \lambda\right)_{\alpha}\left(w \gamma_{m} \bar{\lambda}\right),
$$

where $\lambda^{\alpha}$ and $\mu_{\alpha}$ are constrained to satisfy

$$
\lambda \gamma^{m n} \mu=\mu \gamma^{m} \mu=\lambda \gamma^{m} \lambda=0
$$

The variables $w_{\alpha}$ and $\nu^{\alpha}$ in (3.4) are the conjugate momenta to $\lambda^{\alpha}$ and $\mu_{\alpha}$ and are defined up to the gauge transformations

$$
\delta w_{\alpha}=f_{m}\left(\gamma^{m} \lambda\right)_{\alpha}, \quad \delta \nu^{\alpha}=c_{m n}\left(\gamma^{m n} \lambda\right)^{\alpha}+h_{m}\left(\gamma^{m} \mu\right)^{\alpha},
$$

for arbitrary parameters $c_{m n}, h_{m}$ and $f_{m}$. Note that $\left(\lambda^{\alpha}, \mu_{\alpha}\right)$ satisfying (3.5) contain 16 independent components and can be interpreted as $d=10$ twistor variables. As discussed in $[24,25]$, twistors in $d$ spacetime dimensions are pure spinors in $d+2$ dimensions which transform covariantly under $\mathrm{SO}(d, 2)$ conformal transformations. The spinors $\left(\lambda^{\alpha}, \mu_{\alpha}\right)$ satisfying (3.5) can therefore be interpreted as the 16 independent components of a $\mathrm{d}=12$ pure spinor $U^{A}$ satisfying the pure spinor condition $U^{A} \gamma_{A B}^{M N} U^{B}=0$ where $A=1$ to $32, M=0$ to 11 , and $\gamma^{M}$ are the $d=12$ gamma-matrices. So (3.4) decomposes the 32 components of 
$\Lambda^{\alpha}$ and $\Omega_{\alpha}^{\prime}$ into the 16 independent components of $\left(\lambda^{\alpha}, \mu_{\alpha}\right)$ describing a $\mathrm{d}=12$ pure spinor, and the 16 gauge-invariant components of its conjugate momenta $\left(w_{\alpha}, \nu^{\alpha}\right)$.

For the heterotic superstring, $\Phi_{\alpha}^{\prime}$ does not have enough degrees of freedom to solve for $X^{m}$, but for the Type II superstring, one also has the right-moving superfields

$$
\widehat{\Theta}^{\hat{\alpha}}=\widehat{\theta}^{\hat{\alpha}}+\bar{\kappa} \widehat{\Lambda}^{\hat{\alpha}}, \quad \widehat{\Phi}_{\hat{\alpha}}^{\prime}=\widehat{\Omega}_{\hat{\alpha}}^{\prime}+\bar{\kappa} \widehat{h}_{\hat{\alpha}}^{\prime},
$$

with component expansions

$$
\widehat{\Lambda}^{\hat{\alpha}}=\widehat{\lambda}^{\alpha}+\frac{1}{2(\widehat{\lambda} \hat{\bar{\lambda}})}\left(\gamma^{m} \hat{\bar{\lambda}}\right)^{\hat{\alpha}}\left(\widehat{\lambda} \gamma_{m} \widehat{\nu}\right), \quad \widehat{\Omega}_{\hat{\alpha}}^{\prime}=\widehat{\mu}_{\hat{\alpha}}+\widehat{w}_{\hat{\alpha}}-\frac{1}{2(\widehat{\lambda} \hat{\bar{\lambda}})}\left(\gamma^{m} \widehat{\lambda}\right)_{\hat{\alpha}}\left(\widehat{w} \gamma_{m} \widehat{\bar{\lambda}}\right) .
$$

The shifted superfields $\Phi_{\alpha}^{\prime}$ and $\widehat{\Phi}_{\hat{\alpha}}^{\prime}$ are defined by

$$
\Phi_{\alpha}^{\prime}=\Phi_{\alpha}-\frac{1}{2} X^{m}\left(\gamma_{m} D \Theta\right)_{\alpha}, \quad \widehat{\Phi}_{\hat{\alpha}}^{\prime}=\widehat{\Phi}_{\hat{\alpha}}-\frac{1}{2} X^{m}\left(\gamma_{m} \bar{D} \widehat{\Theta}\right)_{\hat{\alpha}},
$$

and no longer satisfy the constraints $\bar{\lambda} \gamma^{m} \Phi^{\prime}=0$ and $\widehat{\bar{\lambda}} \gamma^{m} \widehat{\Phi}^{\prime}=0$. Since the $\Phi_{\alpha}^{\prime}$ and $\widehat{\Phi}_{\hat{\alpha}}^{\prime}$ superfields are related to the $X^{m}$ superfield by

$$
\bar{\lambda} \gamma^{m} \Phi^{\prime}=-\frac{1}{2}\left(\bar{\lambda} \gamma^{m} \gamma^{n} D \Theta\right) X_{n}, \quad \widehat{\bar{\lambda}} \gamma^{m} \widehat{\Phi}^{\prime}=-\frac{1}{2}\left(\widehat{\bar{\lambda}} \gamma^{m} \gamma^{n} \bar{D} \widehat{\Theta}\right) X_{n},
$$

the bosonic spinor variables $\left(\mu_{\alpha}, \lambda^{\alpha}\right)$ and $\left(\widehat{\mu}_{\hat{\alpha}}, \widehat{\lambda}^{\hat{\alpha}}\right)$ in (3.4) and (3.8) are related to the spacetime vector variable $x^{m}$ by the usual twistor relation

$$
\mu_{\alpha}=-\frac{1}{2} x^{m}\left(\gamma_{m} \lambda\right)_{\alpha}, \quad \widehat{\mu}_{\hat{\alpha}}=-\frac{1}{2} x^{m}\left(\gamma_{m} \widehat{\lambda}\right)_{\hat{\alpha}} .
$$

For the Type IIA superstring, the twistor relation of (3.11) can be inverted to solve for $x^{m}$ in terms of $\mu_{\alpha}$ and $\widehat{\mu}^{\alpha}$ as

$$
x^{m}=-\frac{1}{(\lambda \widehat{\lambda})}\left(\widehat{\lambda} \gamma^{m} \mu+\lambda \gamma^{m} \widehat{\mu}\right)
$$

where it is assumed that $\lambda^{\alpha} \widehat{\lambda}_{\alpha} \neq 0$. Similarly, the Type IIA superfield $X^{m}$ can be expressed in terms of $\Phi_{\alpha}^{\prime}$ and $\widehat{\Phi}^{\prime \alpha}$ as

$$
X^{m}=-\frac{1}{(D \Theta \bar{D} \widehat{\Theta})}\left(\bar{D} \widehat{\Theta} \gamma^{m} \Phi^{\prime}+D \Theta \gamma^{m} \widehat{\Phi}^{\prime}\right) .
$$

Although there is no analogous solution for the uncompactified Type IIB superstring, one can use the standard T-duality relation of Type IIB with Type IIA to solve for $x^{m}$ if at least one direction of the Type IIB superstring is compactified on a circle. For example, if $x^{9}$ is compactified on a circle, define

$$
\mu_{\alpha}=-\frac{1}{2} \widetilde{x}^{m}\left(\gamma_{m} \lambda\right)_{\alpha}, \quad \widehat{\mu}_{\alpha}=-\frac{1}{2} \widetilde{x}^{m}\left(\gamma^{9} \gamma_{m} \gamma_{9} \widehat{\lambda}\right)_{\alpha},
$$

where $\widetilde{x}^{m}$ is the T-dual to $x^{m}$ defined by

$$
\widetilde{x}^{m}=x_{L}^{m}+x_{R}^{m} \text { for } m=0 \text { to } 8, \quad \widetilde{x}^{9}=x_{L}^{9}-x_{R}^{9},
$$


and $x_{L}^{m}$ and $x_{R}^{m}$ are the left and right-moving parts of $x^{m}$ defined by $x_{L}^{m}(z)=\int{ }^{z} d y \partial x^{m}(y)$ and $x_{R}^{m}(\bar{z})=\int^{\bar{z}} d \bar{y} \bar{\partial} x^{m}(\bar{y})$. Using (3.14), one can invert to solve for $\widetilde{x}^{m}$ in terms of $\mu_{\alpha}$ and $\widehat{\mu}_{\alpha}$ as

$$
\widetilde{x}^{m}=-\frac{1}{\left(\lambda \gamma^{9} \widehat{\lambda}\right)}\left(\widehat{\lambda} \gamma^{9} \gamma^{m} \mu+\lambda \gamma^{m} \gamma^{9} \widehat{\mu}\right)
$$

where it is assumed that $\left(\lambda \gamma^{9} \widehat{\lambda}\right) \neq 0$.

\section{$3.2 \quad \mathrm{~N}=1$ superconformal and $\mathrm{U}(1)$ generator}

The left-moving $\mathrm{N}=1$ superconformal stress tensor for the twistor-string is $\mathcal{T}=\frac{1}{2} D \Phi_{\alpha}^{\prime} D \Theta^{\alpha}-$ $\frac{1}{2} \Phi_{\alpha}^{\prime} \partial \Theta^{\alpha}$, which in components is

$$
G=h_{\alpha}^{\prime} \Lambda^{\alpha}-\Omega_{\alpha}^{\prime} \partial \theta^{\alpha}, \quad T=-\frac{1}{2} \Omega_{\alpha}^{\prime} \partial \Lambda^{\alpha}+\frac{1}{2} \partial \Omega_{\alpha}^{\prime} \Lambda^{\alpha}-h_{\alpha}^{\prime} \partial \theta^{\alpha} .
$$

As in the other worldsheet supersymmetric descriptions of the superstring, physical states will be required to be $\mathrm{N}=1$ superconformal primary fields whose integrated vertex operators have zero or negative charge with respect to a $\mathrm{U}(1)$ generator $J$. In the twistor-string description, the $\mathrm{U}(1)$ generator will be defined as

$$
J=-\lambda^{\alpha} w_{\alpha}+\mu_{\alpha} \nu^{\alpha}
$$

which splits $G$ into

$$
G^{+}=h_{\alpha}^{\prime} \lambda^{\alpha}-\mu_{\alpha} \partial \theta^{\alpha}, \quad G^{-}=\frac{1}{2(\lambda \bar{\lambda})}\left(h^{\prime} \gamma_{m} \bar{\lambda}\right)\left(\lambda \gamma^{m} \nu\right)-w_{\alpha} \partial \theta^{\alpha}+\frac{1}{2(\lambda \bar{\lambda})}\left(w \gamma_{m} \bar{\lambda}\right)\left(\lambda \gamma^{m} \partial \theta\right)
$$

where $\left(\lambda^{\alpha}, \mu_{\alpha}, w_{\alpha}, \nu^{\alpha}\right)$ are defined in (3.4). Note that the $\mathrm{U}(1)$ generator $J$ of (3.18) counts the projective weight of the $\mathrm{d}=10$ twistor variables where $\left(\lambda^{\alpha}, \mu_{\alpha}\right)$ carry projective weight +1 and $\left(w_{\alpha}, \nu^{\alpha}\right)$ carry projective weight -1 . So the integrated vertex operator $G V$ will be required to carry zero or negative projective weight, and the term $(G V)_{0}$ of zero projective weight will be required to be globally defined on pure spinor space, i.e. independent of $\bar{\lambda}_{\alpha}$ and invariant under the gauge transformations of (3.6).

\subsection{Worldsheet action in a flat background}

Under spacetime supersymmetry, (3.10) and $\delta X^{m}=-\frac{1}{2}\left(\epsilon \gamma^{m} \Theta+\widehat{\epsilon} \gamma^{m} \widehat{\Theta}\right)$ implies that the $\Phi_{\alpha}^{\prime}$ and $\widehat{\Phi}_{\hat{\alpha}}^{\prime}$ superfields transform as

$$
\begin{array}{ll}
\delta \Theta^{\alpha}=\epsilon^{\alpha}, & \delta \widehat{\Theta}^{\hat{\alpha}}=\widehat{\epsilon}^{\hat{\alpha}}, \\
\delta \Phi_{\alpha}^{\prime}=\frac{1}{4}\left(\epsilon \gamma^{m} \Theta+\widehat{\epsilon} \gamma^{m} \widehat{\Theta}\right)\left(\gamma_{m} D \Theta\right), & \delta \widehat{\Phi}_{\hat{\alpha}}^{\prime}=\frac{1}{4}\left(\epsilon \gamma^{m} \Theta+\widehat{\epsilon} \gamma^{m} \widehat{\Theta}\right)\left(\gamma_{m} \bar{D} \widehat{\Theta}\right) .
\end{array}
$$

And under spacetime translations, $\delta X^{m}=c^{m}$ implies that the $\Phi_{\alpha}^{\prime}$ and $\widehat{\Phi}_{\hat{\alpha}}^{\prime}$ superfields transform as

$$
\delta \Theta^{\alpha}=\delta \widehat{\Theta}^{\hat{\alpha}}=0, \quad \delta \Phi_{\alpha}^{\prime}=-\frac{1}{2} c^{m}\left(\gamma_{m} D \Theta\right), \quad \delta \widehat{\Phi}_{\hat{\alpha}}^{\prime}=-\frac{1}{2} c^{m}\left(\gamma_{m} \bar{D} \widehat{\Theta}\right) .
$$


The $\mathrm{N}=(1,1)$ worldsheet supersymmetric action for the Type II twistor-string in a flat background should be invariant under these super-Poincaré transformations and will be defined in terms of the $\left(\Theta^{\alpha}, \widehat{\Theta}^{\hat{\alpha}}, \Phi_{\alpha}^{\prime}, \widehat{\Phi}_{\hat{\alpha}}^{\prime}\right)$ superfields as

$$
S=\int d^{2} z d^{2} \kappa\left[-\Phi_{\alpha}^{\prime} \bar{D} \Theta+\widehat{\Phi}_{\hat{\alpha}}^{\prime} D \widehat{\Theta}-\frac{1}{8}\left(\Theta \gamma^{m} D \Theta\right)\left(\widehat{\Theta} \gamma_{m} \bar{D} \widehat{\Theta}\right)+\frac{1}{8}\left(\widehat{\Theta} \gamma^{m} D \widehat{\Theta}\right)\left(\Theta \gamma_{m} \bar{D} \Theta\right)\right]
$$

After integrating out auxiliary variables and shifting $w_{\alpha}$ and $\widehat{w}_{\hat{\alpha}},(3.22)$ reduces to

$$
\begin{aligned}
S= & \int d^{2} z\left[h_{\alpha}^{\prime} \bar{\partial} \theta^{\alpha}+\widehat{h}_{\hat{\alpha}}^{\prime} \partial \widehat{\theta}^{\hat{\alpha}}+w_{\alpha} \bar{\partial} \lambda^{\alpha}+\widehat{w}_{\hat{\alpha}} \partial \widehat{\lambda}^{\hat{\alpha}}+\mu_{\alpha} \bar{\partial} \nu^{\alpha}+\widehat{\mu}_{\hat{\alpha}} \partial \widehat{\nu}^{\hat{\alpha}}\right. \\
& \left.-\frac{1}{2}\left(\nu \gamma^{m} \lambda-\frac{1}{2} \theta \gamma^{m} \partial \theta\right)\left(\widehat{\nu} \gamma_{m} \widehat{\lambda}-\frac{1}{2} \widehat{\theta} \gamma_{m} \bar{\partial} \hat{\theta}\right)\right]
\end{aligned}
$$

with the spacetime supersymmetry generators

$$
\begin{aligned}
q_{\alpha} & =\int d z d \kappa \Phi_{\alpha}^{\prime}+\frac{1}{4} \int d \bar{z} d \bar{\kappa}\left(\gamma^{m} \Theta\right)_{\alpha}\left(\widehat{\Theta} \gamma_{m} \bar{D} \widehat{\Theta}\right) \\
& =\int d z h_{\alpha}^{\prime}-\frac{1}{2} \int d \bar{z}\left(\widehat{\lambda} \gamma^{m} \widehat{\nu}-\frac{1}{2} \widehat{\theta} \gamma^{m} \bar{\partial} \widehat{\theta}\right)\left(\gamma_{m} \theta\right)_{\alpha}, \\
\widehat{q}_{\hat{\alpha}} & =\frac{1}{4} \int d z d \kappa\left(\gamma^{m} \widehat{\Theta}\right)_{\hat{\alpha}}\left(\Theta \gamma_{m} D \Theta\right)+\int d \bar{z} d \bar{\kappa}_{\hat{\alpha}}^{\prime} \\
& =-\frac{1}{2} \int d z\left(\lambda \gamma^{m} \nu-\frac{1}{2} \theta \gamma^{m} \partial \theta\right)\left(\gamma_{m} \widehat{\theta}\right)_{\hat{\alpha}}+\int d \bar{z} \widehat{h}_{\hat{\alpha}}^{\prime} \\
P_{m} & =\frac{1}{2} \int d z d \kappa \Theta \gamma_{m} D \Theta+\frac{1}{2} \int d \bar{z} d \bar{\kappa} \widehat{\Theta} \gamma_{m} \bar{D} \widehat{\Theta} \\
& =\int d z\left(\lambda \gamma_{m} \nu-\frac{1}{2} \theta \gamma_{m} \partial \theta\right)+\int d \bar{z}\left(\widehat{\lambda} \gamma_{m} \widehat{\nu}-\frac{1}{2} \widehat{\theta} \gamma_{m} \bar{\partial} \widehat{\theta}\right)
\end{aligned}
$$

Using that $\Phi_{\alpha}^{\prime}+\frac{1}{2} X^{m}\left(\gamma_{m} D \Theta\right)_{\alpha}$ and $\widehat{\Phi}_{\hat{\alpha}}^{\prime}+\frac{1}{2} X^{m}\left(\gamma_{m} \bar{D} \widehat{\Theta}\right)_{\hat{\alpha}}$ are spacetime supersymmetric, the action of (3.22) can be written in manifestly spacetime supersymmetric notation as

$$
S=\int d^{2} z d^{2} \kappa\left[-\left(\Phi_{\alpha}^{\prime}+\frac{1}{2} X^{m}\left(\gamma_{m} D \Theta\right)_{\alpha}\right) \bar{D} \Theta^{\alpha}+\left(\widehat{\Phi}_{\hat{\alpha}}^{\prime}+\frac{1}{2} X^{m}\left(\gamma_{m} \bar{D} \widehat{\Theta}\right)_{\hat{\alpha}}\right) D \widehat{\Theta}^{\hat{\alpha}}+B_{\kappa \bar{\kappa}}^{I I}\right]
$$

where $B_{\kappa \bar{\kappa}}^{I I}$ is defined in (2.26). Note that this action is related to the Type II worldsheet action of (2.22) by dropping the term

$$
\frac{1}{2} \int d^{2} z d \kappa d \bar{\kappa} \Pi_{\kappa}^{m} \bar{\Pi}_{\bar{\kappa} m}
$$

Since $\Pi_{\kappa}^{m} \bar{\Pi}_{\bar{\kappa} m}$ has left and right-moving $\mathrm{U}(1)$ charge $(-1,-1)$, the term of zero U(1) charge in (3.26) can be expressed as the BRST-trivial term

$$
-\frac{1}{2} \int d^{2} z \oint G^{+} \oint \widehat{G}^{+}\left(\Pi_{\kappa}^{m} \bar{\Pi}_{\bar{\kappa} m}\right) .
$$

So if all vertex operators are annihilated by $\oint G^{+}$and $\oint \widehat{G}^{+}$, it seems reasonable to assume that dropping the term of (3.27) will not affect the scattering amplitudes since one can pull 
the countour integrals of $G^{+}$and $\widehat{G}^{+}$off of the surface. However, since vertex operators have not yet been constructed in the twistor-string formalism, this assumption has not yet been verified by explicit computations.

To relate (3.22) with the usual component form of the Type IIA pure spinor worldsheet action, substitute $\mu_{\alpha}=-\frac{1}{2} x^{m}\left(\gamma_{m} \lambda\right)_{\alpha}$ and $\widehat{\mu}^{\alpha}=-\frac{1}{2} x^{m}\left(\gamma_{m} \widehat{\lambda}\right)^{\alpha}$ into (3.23) and vary $\nu^{\alpha}$ and $\widehat{\nu}_{\alpha}$ to obtain the equations of motion

$$
\left(\widehat{\lambda} \gamma^{m} \widehat{\nu}\right)\left(\gamma_{m} \lambda\right)_{\alpha}=\bar{\pi}^{m}\left(\gamma_{m} \lambda\right)_{\alpha}, \quad\left(\lambda \gamma^{m} \nu\right)\left(\gamma_{m} \widehat{\lambda}\right)^{\alpha}=\pi^{m}\left(\gamma_{m} \widehat{\lambda}\right)^{\alpha},
$$

which implies

$$
\left(\widehat{\lambda} \gamma^{m} \widehat{\nu}\right)=\frac{\left(\widehat{\lambda} \gamma^{m} \gamma^{n} \lambda\right)}{2 \lambda \widehat{\lambda}} \bar{\pi}_{n}, \quad\left(\lambda \gamma^{m} \nu\right)=\frac{\left(\lambda \gamma^{m} \gamma^{n} \widehat{\lambda}\right)}{2 \lambda \widehat{\lambda}} \pi_{n}
$$

where the equations of motion $\bar{\partial} \lambda^{\alpha}=\partial \widehat{\lambda}_{\alpha}=0$ have been used. Plugging the auxiliary equations of (3.29) back into the action of (3.23) and ignoring terms which vanish when $\bar{\partial} \lambda^{\alpha}=\partial \widehat{\lambda}_{\alpha}=0$ (and can therefore be cancelled by an appropriate shift of $w_{\alpha}$ and $\widehat{w}^{\alpha}$ ), one finds that

$$
\begin{aligned}
S= & \int d^{2} z\left[h_{\alpha}^{\prime} \bar{\partial} \theta^{\alpha}+\widehat{h}_{\hat{\alpha}}^{\prime} \partial \widehat{\theta}^{\hat{\alpha}}+w_{\alpha} \bar{\partial} \lambda^{\alpha}+\widehat{w}_{\hat{\alpha}} \partial \widehat{\lambda}^{\hat{\alpha}}\right. \\
& \left.+\frac{1}{2} \pi^{m} \bar{\pi}_{m}-\frac{\left(\lambda \gamma^{m} \gamma^{n} \widehat{\lambda}\right)}{2 \lambda \widehat{\lambda}} \pi_{m} \bar{\pi}_{n}-\frac{1}{8}\left(\theta \gamma_{m} \partial \theta\right)\left(\widehat{\theta} \gamma^{m} \bar{\partial} \widehat{\theta}\right)\right] \\
= & \int d^{2} z\left[p_{\alpha} \bar{\partial} \theta^{\alpha}+\widehat{p}_{\hat{\alpha}} \partial \widehat{\theta}^{\hat{\alpha}}+w_{\alpha} \bar{\partial} \lambda^{\alpha}+\widehat{w}_{\hat{\alpha}} \partial \widehat{\lambda}^{\hat{\alpha}}+\frac{1}{2} \partial x^{m} \bar{\partial} x_{m}\right] \\
& -\int d^{2} z \frac{\left(\lambda \gamma^{m} \gamma^{n} \widehat{\lambda}\right)}{2 \lambda \widehat{\lambda}} \pi_{m} \bar{\pi}_{n},
\end{aligned}
$$

where

$$
\begin{aligned}
& p_{\alpha}=h_{\alpha}^{\prime}+\frac{1}{2} x_{m}\left(\gamma^{m} \partial \theta\right)_{\alpha}+\frac{1}{2}\left(\partial x_{m}+\frac{1}{4} \theta \gamma_{m} \partial \theta+\frac{1}{8} \widehat{\theta} \gamma_{m} \partial \widehat{\theta}\right)\left(\gamma^{m} \theta\right)_{\alpha}, \\
& \widehat{p}^{\alpha}=\widehat{h}^{\prime \alpha}+\frac{1}{2} x_{m}\left(\gamma^{m} \bar{\partial} \widehat{\theta}\right)^{\alpha}+\frac{1}{2}\left(\bar{\partial} x_{m}+\frac{1}{4} \widehat{\theta} \gamma_{m} \bar{\partial} \widehat{\theta}+\frac{1}{8} \theta \gamma_{m} \bar{\partial} \theta\right)\left(\gamma^{m} \widehat{\theta}\right)^{\alpha},
\end{aligned}
$$

and the first line of (3.31) is the Type IIA worldsheet action of (2.30). Furthermore, (3.32) implies that $G^{+}$and $\widehat{G}^{+}$of (3.19) are mapped into the pure spinor BRST currents

$$
\begin{aligned}
& G^{+}=\lambda^{\alpha} h_{\alpha}^{\prime}-\mu_{\alpha} \partial \theta^{\alpha} \rightarrow G^{+}=\lambda^{\alpha} d_{\alpha}, \\
& \widehat{G}^{+}=\widehat{\lambda}_{\alpha} \widehat{h}^{\prime \alpha}-\widehat{\mu}^{\alpha} \bar{\partial} \widehat{\theta}_{\alpha} \rightarrow \widehat{G}^{+}=\widehat{\lambda}_{\alpha} \widehat{d}^{\alpha},
\end{aligned}
$$

where

$$
d_{\alpha}=p_{\alpha}-\frac{1}{2}\left(\partial x^{m}+\frac{1}{4} \theta \gamma^{m} \partial \theta\right)\left(\gamma_{m} \theta\right)_{\alpha}, \quad \widehat{d}^{\alpha}=\widehat{p}^{\alpha}-\frac{1}{2}\left(\bar{\partial} x^{m}+\frac{1}{4} \widehat{\theta} \gamma^{m} \bar{\partial} \hat{\theta}\right)\left(\gamma_{m} \widehat{\theta}\right)^{\alpha}
$$

are defined as in (2.3) and the equations of motion $\bar{\partial} \theta^{\alpha}=\partial \widehat{\theta}_{\alpha}=0$ have been used.

Finally, note that

$$
-\int d^{2} z \frac{\left(\lambda \gamma^{m} \gamma^{n} \widehat{\lambda}\right)}{2 \lambda \widehat{\lambda}} \pi_{m} \bar{\pi}_{n}=\int d^{2} z \oint G^{+} \oint \widehat{G}^{+}\left(\frac{1}{2 \lambda \widehat{\lambda}} d_{\alpha} \widehat{d}^{\alpha}\right)
$$

in the second line of (3.31) is BRST-trivial. So up to this BRST-trivial term related to (3.27), the Type II twistor-string action of (3.22) is equal to the pure spinor action of $(2.30)$. 


\subsection{Twistor string in $\mathrm{AdS}_{5} \times S^{5}$ background}

In principle, one can obtain the worldsheet action for the twistor string in an $\mathrm{AdS}_{5} \times$ $S^{5}$ background by deforming the Type IIB action in a flat background with the vertex operator for the Ramond-Ramond five-form field strength and computing the back-reaction. However, a simpler method is to find a $\mathrm{PSU}(2,2 \mid 4)$-invariant action which reduces in the large radius limit to the Type IIB twistor string action in a flat background of (3.22).

To describe the $\mathrm{AdS}_{5} \times S^{5}$ background, it is convenient to start with the standard representation of $\mathrm{AdS}_{5} \times S^{5}$ where $g(Z)$ takes values in the supercoset $\frac{\mathrm{PSU}(2,2 \mid 4)}{\mathrm{SO}(4,1) \times \mathrm{SO}(5)}$ and define the supervierbein $E_{M}^{A}$ by

$$
g^{-1} \partial g=E_{M}^{A} \partial Z^{M}
$$

where $A=(a, \alpha, \hat{\alpha})$ denote the 10 bosonic and 32 fermionic generators of $\frac{\operatorname{PSU}(2,2 \mid 4)}{\operatorname{SO}(4,1) \times \operatorname{SO}(5)}$ and $Z^{M}=\left[x^{m}, \theta^{\mu}, \widehat{\theta}^{\hat{\mu}}\right]$. Using the notation $\eta_{\alpha \hat{\beta}}=\left(\gamma^{01234}\right)_{\alpha \hat{\beta}}$ and $\eta^{\alpha \hat{\beta}}=\left(\gamma_{01234}\right)^{\alpha \hat{\beta}}$, the Ramond-Ramond field strength is given by $F^{\alpha \hat{\beta}}=\eta^{\alpha \hat{\beta}}$ and one can choose the gauge where

$$
\begin{aligned}
B_{M N} D Z^{M} \bar{D} Z^{N} & =\eta_{\alpha \hat{\alpha}}\left(E_{M}^{\alpha} E_{N}^{\hat{\alpha}}+E_{N}^{\alpha} E_{M}^{\hat{\alpha}}\right) D Z^{M} \bar{D} Z^{N} \\
& =\eta_{\alpha \hat{\alpha}}\left(\left(g^{-1} D g\right)^{\alpha}\left(g^{-1} \bar{D} g\right)^{\hat{\alpha}}+\left(g^{-1} D g\right)^{\hat{\alpha}}\left(g^{-1} \bar{D} g\right)^{\alpha}\right) .
\end{aligned}
$$

Just as the term $\frac{1}{2} \int d^{2} z \int d \kappa d \bar{\kappa} \Pi_{\kappa}^{m} \bar{\Pi}_{\bar{\kappa} m}$ of (3.26) was dropped from the twistor-string action in a flat background, the twistor-string action in an $\operatorname{AdS}_{5} \times S^{5}$ background will be defined by dropping the analog of this term in the curved background action of (2.54)

$$
\int d^{2} z \int d \kappa d \bar{\kappa} \frac{1}{2} \eta_{a b} E_{M}^{a} E_{N}^{b} D Z^{M} \bar{D} Z^{N}=\int d^{2} z \int d \kappa d \bar{\kappa} \frac{1}{2} \eta_{a b}\left(g^{-1} D g\right)^{a}\left(g^{-1} \bar{D} g\right)^{b} .
$$

So the twistor-string action is

$$
\begin{aligned}
S= & r^{2} \int d^{2} z d \kappa d \bar{\kappa}\left[\frac{1}{2} \eta_{\alpha \hat{\alpha}}\left(\left(g^{-1} D g\right)^{\alpha}\left(g^{-1} \bar{D} g\right)^{\hat{\alpha}}+\left(g^{-1} D g\right)^{\hat{\alpha}}\left(g^{-1} \bar{D} g\right)^{\alpha}\right)\right. \\
& \left.-\Phi_{\alpha}^{\prime}\left(g^{-1} D g\right)^{\alpha}+\widehat{\Phi}_{\hat{\alpha}}^{\prime}\left(g^{-1} \bar{D} g\right)^{\hat{\alpha}}-\eta^{\alpha \hat{\alpha}} \Phi_{\alpha}^{\prime} \widehat{\Phi}_{\hat{\alpha}}^{\prime}\right]
\end{aligned}
$$

where $r$ is the $\mathrm{AdS}_{5}$ radius and $X^{m}$ is determined in terms of $\left[\Theta^{\alpha}, \widehat{\Theta}^{\hat{\alpha}}, \Phi_{\alpha}^{\prime}, \widehat{\Phi}_{\hat{\alpha}}^{\prime}\right]$ through the $\mathrm{AdS}_{5} \times S^{5}$ generalization of (3.13).

Since the $\mathrm{AdS}_{5} \times S^{5}$ superstring is a Type IIB superstring, defining $X^{m}$ in terms of $\left[\Theta^{\alpha}, \widehat{\Theta}^{\hat{\alpha}}, \Phi_{\alpha}^{\prime}, \widehat{\Phi}_{\hat{\alpha}}^{\prime}\right]$ will require T-dualizing one of the $\mathrm{AdS}_{5} \times S^{5}$ directions as explained in (3.14). A convenient choice which will hopefully be explored in a future paper is to T-dualize one of the $S^{5}$ directions, which breaks the manifest SU(4) R-symmetry to $\mathrm{SO}(4) \times \mathrm{U}(1)$. This is the same manifest symmetry as the spin-chain, and T-dualizing in this direction means that the spin-chain ground state $\operatorname{Tr}\left(Z^{n}\right)$ where $Z$ is the scalar with $+1 \mathrm{U}(1)$ charge is described by a string with winding number $n$. 
Integrating out the auxiliary superfields $\Phi_{\alpha}^{\prime}$ and $\widehat{\Phi}_{\hat{\alpha}}^{\prime}$ in (3.39), the $\operatorname{AdS}_{5} \times S^{5}$ twistorstring action simplifies to

$$
S=r^{2} \int d^{2} z d \kappa d \bar{\kappa} \frac{1}{2} \eta_{\alpha \hat{\alpha}}\left[\left(g^{-1} D g\right)^{\alpha}\left(g^{-1} \bar{D} g\right)^{\hat{\alpha}}-\left(g^{-1} D g\right)^{\hat{\alpha}}\left(g^{-1} \bar{D} g\right)^{\alpha}\right] .
$$

Surprisingly, this action is not only invariant under the local transformation $\delta g=g \Omega$ when $\Omega \in \mathrm{SO}(4,1) \times \mathrm{SO}(5)$, it is also invariant under $\delta g=g \Omega$ when $\Omega \in \mathrm{SO}(4,2) \times \mathrm{SO}(6)$. So the bosonic elements of the coset can be gauged away and the action of (3.40) can be expressed as

$$
S=r^{2} \int d^{2} z d \kappa d \bar{\kappa} \frac{1}{2} \eta_{\alpha \hat{\alpha}}\left[\left(G^{-1} D G\right)^{\alpha}\left(G^{-1} \bar{D} G\right)^{\hat{\alpha}}-\left(G^{-1} D G\right)^{\hat{\alpha}}\left(G^{-1} \bar{D} G\right)^{\alpha}\right]
$$

where $G$ is a fermionic coset taking values in $\frac{\mathrm{PSU}(2,2 \mid 4)}{\mathrm{SO}(4,2) \times \mathrm{SO}(6)} \cdot{ }^{2}$

Using $\mathrm{SU}(2,2) \times \mathrm{SU}(4)$ notation, this action can be expressed as

$$
S=r^{2} \int d^{2} z d \kappa d \bar{\kappa}\left(G^{-1} D G\right)_{R}^{J}\left(G^{-1} \bar{D} G\right)_{J}^{R}
$$

where $J=1$ to 4 is a spinor representation of $\mathrm{SO}(4,2)=\mathrm{SU}(2,2)$ and $R=1$ to 4 is a spinor representation of $\mathrm{SO}(6)=\mathrm{SU}(4)$. Note that the Maurer-Cartan equations imply that

$$
\int d^{2} z d \kappa d \bar{\kappa}\left(G^{-1} D G\right)_{R}^{J}\left(G^{-1} \bar{D} G\right)_{J}^{R}=-\int d^{2} z d \kappa d \bar{\kappa}\left(G^{-1} D G\right)_{J}^{R}\left(G^{-1} \bar{D} G\right)_{R}^{J}
$$

up to a surface term. Defining the coset representative of $G$ as

$$
G=e^{\Theta_{R}^{J} T_{J}^{R}} e^{\tilde{\Theta}_{J}^{R} T_{R}^{J}}
$$

where $T_{R}^{J}$ and $T_{J}^{R}$ are the generators of the 32 fermionic isometries, the left-invariant forms are

$$
\left(G^{-1} \partial G\right)_{R}^{J}=\partial \Theta_{R}^{J}, \quad\left(G^{-1} \partial G\right)_{J}^{R}=\partial \tilde{\Theta}_{J}^{R}+\tilde{\Theta}_{K}^{R} \partial \Theta_{S}^{K} \tilde{\Theta}_{J}^{S},
$$

and under the 32 global fermionic isometries generated by $\delta G=\left(\epsilon_{R}^{J} T_{J}^{R}+\tilde{\epsilon}_{J}^{R} T_{R}^{J}\right) G$, the superfields of (3.44) transform as

$$
\delta \Theta_{R}^{J}=\epsilon_{R}^{J}+\Theta_{S}^{J} \tilde{\epsilon}_{K}^{S} \Theta_{R}^{K}, \quad \delta \tilde{\Theta}_{J}^{R}=\tilde{\epsilon}_{J}^{R}-\tilde{\epsilon}_{K}^{R} \Theta_{S}^{K} \tilde{\Theta}_{J}^{S}-\tilde{\Theta}_{K}^{R} \Theta_{S}^{K} \tilde{\epsilon}_{J}^{S} .
$$

Plugging in the left-invariant forms of (3.45) into the action of (3.42), one obtains the remarkably simple action

$$
S=r^{2} \int d^{2} z d \kappa d \bar{\kappa}\left(D \Theta_{R}^{J} \bar{D} \tilde{\Theta}_{J}^{R}+D \Theta_{R}^{J} \tilde{\Theta}_{K}^{R} \bar{D} \Theta_{S}^{K} \tilde{\Theta}_{J}^{S}\right)
$$

To show that the action of (3.47) reduces in the large radius limit to the flat space action of (3.22), rescale

$$
\Theta_{R}^{J} \rightarrow \frac{1}{\sqrt{r}} \Theta_{R}^{J}, \quad \tilde{\Theta}_{J}^{R} \rightarrow \frac{1}{\sqrt{r}} \tilde{\Theta}_{J}^{R}
$$

\footnotetext{
${ }^{2}$ This action has vanishing beta function since the coset is a symmetric space with $\operatorname{PSU}(2,2 \mid 4)$ in the numerator and there is no WZW term [26].
} 
and write (3.47) as

$$
S=\int d^{2} z d \kappa d \bar{\kappa}\left[-\Phi_{\alpha}^{\prime} \bar{D} \Theta^{\alpha}+\widehat{\Phi}_{\hat{\alpha}}^{\prime} D \widehat{\Theta}^{\hat{\alpha}}-\frac{1}{r} \eta^{\alpha \hat{\alpha}} \Phi_{\alpha}^{\prime} \widehat{\Phi}_{\hat{\alpha}}^{\prime}+D \Theta_{R}^{J} \tilde{\Theta}_{K}^{R} \bar{D} \Theta_{S}^{K} \tilde{\Theta}_{J}^{S}\right]
$$

where in terms of the $\Theta^{\alpha}$ and $\widehat{\Theta}^{\hat{\alpha}}$ superfields of (3.39) written in $\mathrm{d}=10$ spinor notation with $\alpha, \hat{\alpha}=1$ to $16, \Theta_{R}^{J}$ only involves the linear combination $\Theta^{\alpha}+i \widehat{\Theta}^{\hat{\alpha}}$ and $\tilde{\Theta}_{J}^{R}$ only involves the linear combination $\Theta^{\alpha}-i \widehat{\Theta}^{\hat{\alpha}}$. After expressing the quartic term in (3.49) in terms of $\Theta^{\alpha}$ and $\widehat{\Theta}^{\hat{\alpha}}$,

$$
\begin{aligned}
S= & \int d^{2} z d \kappa d \bar{\kappa}\left[-\Phi_{\alpha}^{\prime} D \Theta^{\alpha}+\widehat{\Phi}_{\hat{\alpha}}^{\prime} \bar{D} \widehat{\Theta}^{\hat{\alpha}}-\frac{1}{r} \eta^{\alpha \hat{\alpha}} \Phi_{\alpha}^{\prime} \widehat{\Phi}_{\hat{\alpha}}^{\prime}\right. \\
& +e\left(D \Theta \gamma^{a} \Theta+D \widehat{\Theta} \gamma^{a} \widehat{\Theta}\right)\left(\bar{D} \Theta \gamma_{a} \Theta+\bar{D} \widehat{\Theta} \gamma_{a} \widehat{\Theta}\right) \\
& \left.+f_{a b c d e f}\left(D \Theta \gamma^{a b c} \Theta+D \widehat{\Theta} \gamma^{a b c} \widehat{\Theta}\right)\left(\bar{D} \Theta \gamma^{\operatorname{def}} \Theta+\bar{D} \widehat{\Theta} \gamma^{\text {def }} \widehat{\Theta}\right)\right]
\end{aligned}
$$

where $e$ and $f_{\text {abcdef }}$ are constants which are invariant under $\mathrm{SO}(4,1) \times \mathrm{SO}(5)$ transformations and come from expressing $D \Theta_{R}^{J} \tilde{\Theta}_{K}^{R} \bar{D} \Theta_{S}^{K} \tilde{\Theta}_{J}^{S}$ in $\mathrm{d}=10$ notation. When $r \rightarrow \infty$, the $\eta^{\alpha \hat{\alpha}} \Phi_{\alpha}^{\prime} \widehat{\Phi}_{\hat{\alpha}}^{\prime}$ term drops out of (3.50) and, after appropriately shifting $\Phi_{\alpha}^{\prime}$ and $\widehat{\Phi}_{\hat{\alpha}}^{\prime}$ to cancel terms proportional to $\bar{D} \Theta^{\alpha}$ and $D \widehat{\Theta}^{\hat{\alpha}}$, the quartic terms in (3.50) can be reduced to

$$
e\left[\left(D \Theta \gamma^{a} \Theta\right)\left(\bar{D} \widehat{\Theta} \gamma_{a} \widehat{\Theta}\right)-\left(D \widehat{\Theta} \gamma^{a} \widehat{\Theta}\right)\left(\bar{D} \Theta \gamma_{a} \Theta\right)\right]
$$

Finally, the coefficient $e$ can be scaled to $-\frac{1}{8}$ by scaling $\left[\Phi_{\alpha}^{\prime}, \Theta^{\alpha}, \widehat{\Phi}_{\hat{\alpha}}^{\prime}, \widehat{\Theta}^{\hat{\alpha}}\right]$ appropriately so that the $\mathrm{AdS}_{5} \times S^{5}$ action reduces to the flat space action of (3.22).

\section{5 $U(1)$ generator and $d=12$ pure spinors}

Expanding in components, the action of (3.47) is

$$
\begin{aligned}
S= & r^{2} \int d^{2} z\left[\left(G^{-1} \partial G\right)_{R}^{J}\left(G^{-1} \bar{\partial} G\right)_{J}^{R}\right. \\
& \left.+\Lambda_{R}^{J}(\bar{\nabla} \tilde{\Lambda})_{J}^{R}+\widehat{\Lambda}_{R}^{J}(\nabla \tilde{\widehat{\Lambda}})_{J}^{R}+\Lambda_{R}^{J} \tilde{\Lambda}_{K}^{R} \widehat{\Lambda}_{S}^{K} \tilde{\widehat{\Lambda}}_{J}^{S}-\tilde{\Lambda}_{J}^{R} \Lambda_{S}^{J} \tilde{\widehat{\Lambda}}_{K}^{S} \widehat{\Lambda}_{R}^{K}\right]
\end{aligned}
$$

where the bosonic components are defined by

$$
\begin{aligned}
\Lambda_{R}^{J} & =\left.\left(G^{-1} D G\right)_{R}^{J}\right|_{\kappa=\bar{\kappa}=0}, \quad \tilde{\Lambda}_{J}^{R}=\left.\left(G^{-1} D G\right)_{J}^{R}\right|_{\kappa=\bar{\kappa}=0}, \\
\widehat{\Lambda}_{R}^{J} & =\left.\left(G^{-1} \bar{D} G\right)_{R}^{J}\right|_{\kappa=\bar{\kappa}=0}, \quad \tilde{\widehat{\Lambda}}_{J}^{R}=\left.\left(G^{-1} \bar{D} G\right)_{J}^{R}\right|_{\kappa=\bar{\kappa}=0}, \\
(\bar{\nabla} \tilde{\Lambda})_{J}^{R} & =\bar{\partial} \tilde{\Lambda}_{J}^{R}+\left(G^{-1} \bar{\partial} G\right)_{S}^{R} \tilde{\Lambda}_{J}^{S}-\tilde{\Lambda}_{K}^{R}\left(G^{-1} \bar{\partial} G\right)_{J}^{K}, \\
(\nabla \tilde{\hat{\Lambda}})_{J}^{R} & =\partial \tilde{\Lambda}_{J}^{R}+\left(G^{-1} \partial G\right)_{S}^{R} \tilde{\Lambda}_{J}^{S}-\tilde{\Lambda}_{K}^{R}\left(G^{-1} \partial G\right)_{J}^{K} .
\end{aligned}
$$

In order to construct the $\mathrm{U}(1)$ generator needed to define physical states, it is useful to combine the $\mathrm{SO}(4,2) \times \mathrm{SO}(6)$ spinors $\left(G^{-1} \partial G\right)_{R}^{J}$ and $\left(G^{-1} \partial G\right)_{J}^{R}$ into an $\mathrm{SO}(10,2)$ spinor 
$\left(G^{-1} \partial G\right)^{A}$ for $A=1$ to 32 and write (3.52) in $\mathrm{SO}(10,2)$ notation as

$$
\begin{aligned}
S= & r^{2} \int d^{2} z d^{2} \kappa \epsilon_{A B}\left(G^{-1} D G\right)^{A}\left(G^{-1} \bar{D} G\right)^{B} \\
= & r^{2} \int d^{2} z\left[\epsilon_{A B}\left(G^{-1} \partial G\right)^{A}\left(G^{-1} \bar{\partial} G\right)^{B}+\epsilon_{A B} \Lambda^{A}(\bar{\nabla} \Lambda)^{B}+\epsilon_{A B} \widehat{\Lambda}^{A}(\nabla \widehat{\Lambda})^{B}\right. \\
& \left.+R_{M N P Q}\left(\Lambda \gamma^{M N} \Lambda\right)\left(\widehat{\Lambda} \gamma^{P Q} \widehat{\Lambda}\right)\right],
\end{aligned}
$$

where $A=1$ to 32 are $\mathrm{d}=12$ spinor indices and $M=0$ to 11 are $\mathrm{d}=12$ vector indices, $\epsilon_{A B}$ is the Lorentz-covariant antisymmetric tensor used to raise and lower $\mathrm{d}=12$ spinor indices, $\gamma^{M}$ are the $\mathrm{d}=12$ gamma matrices, $\gamma_{A B}^{M N}=\gamma_{B A}^{M N}$ are products of gamma matrices, and

$$
\begin{aligned}
& R_{M N P Q}=-\eta_{M P} \eta_{N Q}+\eta_{N P} \eta_{M Q} \quad \text { when } \quad 0 \leq M, N, P, Q \leq 5, \\
& R_{M N P Q}=\eta_{M P} \eta_{N Q}-\eta_{N P} \eta_{M Q} \quad \text { when } \quad 6 \leq M, N, P, Q \leq 11 .
\end{aligned}
$$

As in a flat background, a U(1) generator $J$ can be defined which splits $G=G^{+}+G^{-}$ where $G^{ \pm}$carries $\pm 1 \mathrm{U}(1)$ charge. This $\mathrm{U}(1)$ generator is constructed by first splitting the bosonic components $\Lambda^{A}$ and $\widehat{\Lambda}^{B}$ of (3.55) into left and right-moving $d=(10,2)$ pure spinors $U^{A}$ and $\widehat{U}^{A}$ satisfying the constraints

$$
U^{A}\left(\gamma^{M N}\right)_{A B} U^{B}=0, \quad \widehat{U}^{A}\left(\gamma^{M N}\right)_{A B} \widehat{U}^{B}=0,
$$

together with their conjugate momenta $V_{A}$ and $\widehat{V}_{A}$. One then defines the left and rightmoving $\mathrm{U}(1)$ generators as

$$
J=-U^{A} V_{A}, \quad \bar{J}=-\widehat{U}^{A} \widehat{V}_{A},
$$

so that $U^{A}$ and $\widehat{U}^{A}$ carry +1 charge and $V_{A}$ and $\widehat{V}_{A}$ carry -1 charge.

Just as a $d=10$ pure spinor parameterizes $\frac{\mathrm{SO}(10)}{\mathrm{U}(5)} \times C$ and has 11 independent complex components, a $d=(10,2)$ pure spinor parameterizes $\frac{\mathrm{SO}(10,2)}{\mathrm{U}(5,1)} \times C$ and has 16 independent complex components. So $J$ splits the 32 components of $\Lambda^{A}$ into the 16 components of $U^{A}$ and 16 components of $V_{A}$. To relate $\Lambda^{A}$ with $U^{A}$ and $V_{A}$, introduce a fixed $d=(10,2)$ pure spinor $\bar{U}_{A}$ on the patch of pure spinor space where $U^{A} \bar{U}_{A} \neq 0$, and define

$$
\Lambda^{A}=U^{A}+\epsilon^{A B} V_{B}+\frac{1}{(U \bar{U})}\left[\frac{1}{4}\left(\gamma^{M N} U\right)^{A}\left(\bar{U} \gamma_{M N} V\right)+2 U^{A}(\bar{U} V)\right],
$$

where the coefficients in (3.59) have been chosen such that $\bar{U} \gamma^{M N} \Lambda=\bar{U} \gamma^{M N} U$. Note that (3.59) is invariant under the gauge transformation

$$
\delta V_{A}=\Omega^{M N}\left(\gamma_{M N} U\right)_{A}
$$

for any $\Omega^{M N}$, which allows 16 components of $V_{A}$ to be gauged to zero.

When written in terms of $\left(U^{A}, V_{A}\right)$ and $\left(\widehat{U}^{A}, \widehat{V}_{A}\right)$, the worldsheet action of (3.55) has no terms with positive $\mathrm{U}(1)$ charge and the term with zero $\mathrm{U}(1)$ charge is

$$
\begin{aligned}
S= & r^{2} \int d^{2} z\left[\epsilon_{A B}\left(G^{-1} \partial G\right)^{A}\left(G^{-1} \bar{\partial} G\right)^{B}+V_{A}(\bar{\nabla} U)^{A}+\widehat{V}_{A}(\nabla \widehat{U})^{A}\right. \\
& \left.+R_{M N P Q}\left(V \gamma^{M N} U\right)\left(\widehat{V} \gamma^{P Q} \widehat{U}\right)\right]
\end{aligned}
$$


where $R_{M N P Q}$ is defined in (3.56). As expected, the term of zero U(1) charge in (3.55) is independent of the fixed pure spinors $\bar{U}$ and $\bar{U}$ and gauge-invariant under (3.60).

Under the $\mathrm{SO}(4,2) \times \mathrm{SO}(6)$ subgroup of $\mathrm{SO}(10,2), U^{A}$ decomposes into $\left(\tilde{U}_{J}^{R}, U_{R}^{J}\right)$ and the $d=(10,2)$ pure spinor constraint of $(3.57)$ decomposes as

$$
\begin{aligned}
\tilde{U}_{J}^{R} U_{S}^{J} & =\frac{1}{4} \delta_{S}^{R} \tilde{U}_{J}^{T} U_{T}^{J}, & U_{R}^{J} \tilde{U}_{K}^{R} & =\frac{1}{4} \delta_{K}^{J} U_{R}^{L} \tilde{U}_{L}^{R}, \\
\epsilon^{J K L M} \tilde{U}_{J}^{R} \tilde{U}_{K}^{S} & =\epsilon^{R S T U} U_{T}^{L} U_{U}^{M}, & \epsilon_{J K L M} U_{R}^{J} U_{S}^{K} & =\epsilon_{R S T U} \tilde{U}_{L}^{T} \tilde{U}_{M}^{U} .
\end{aligned}
$$

Note that the second line of (3.62) is not invariant under the U(1) 'bonus' symmetry which rotates

$$
U_{S}^{J} \rightarrow e^{i \phi} U_{S}^{J}, \quad \tilde{U}_{J}^{S} \rightarrow e^{-i \phi} \tilde{U}_{J}^{S}
$$

and enlarges the R-symmetry group from SU(4) to U(4). So although the action of (3.52) is invariant under this $\mathrm{U}(1)$ bonus symmetry, $J$ of (3.58) is not invariant which implies that the action of (3.61) with zero $\mathrm{U}(1)$ charge is also not invariant under this bonus symmetry.

\section{Acknowledgments}

I would like to thank CNPq grant 300256/94-9 and FAPESP grants 2011/11973-4 and 2014/18634-9 for partial financial support, Nikita Nekrasov and Edward Witten for suggesting to look for an $\mathrm{N}=1$ worldsheet supersymmetric description of the pure spinor formalism, and Andrei Mikhailov, Warren Siegel, Cumrun Vafa and Pedro Vieira for useful discussions.

Open Access. This article is distributed under the terms of the Creative Commons Attribution License (CC-BY 4.0), which permits any use, distribution and reproduction in any medium, provided the original author(s) and source are credited.

\section{References}

[1] N. Berkovits, Super Poincaré covariant quantization of the superstring, JHEP 04 (2000) 018 [hep-th/0001035] [INSPIRE].

[2] H. Gomez and C.R. Mafra, The closed-string 3-loop amplitude and S-duality, JHEP 10 (2013) 217 [arXiv: 1308.6567] [INSPIRE].

[3] L. Mazzucato, Superstrings in AdS, Phys. Rept. 521 (2012) 1 [arXiv:1104.2604] [INSPIRE].

[4] N. Berkovits and N. Nekrasov, Multiloop superstring amplitudes from non-minimal pure spinor formalism, JHEP 12 (2006) 029 [hep-th/0609012] [INSPIRE].

[5] N. Berkovits and E. Witten, Supersymmetry Breaking Effects using the Pure Spinor Formalism of the Superstring, JHEP 06 (2014) 127 [arXiv: 1404.5346] [INSPIRE].

[6] N. Berkovits, Pure spinor formalism as an $N=2$ topological string, JHEP 10 (2005) 089 [hep-th/0509120] [INSPIRE].

[7] E. Witten, Twistor-Like Transform in Ten-Dimensions, Nucl. Phys. B 266 (1986) 245 [INSPIRE]. 
[8] D.P. Sorokin, V.I. Tkach, D.V. Volkov and A.A. Zheltukhin, From the Superparticle Siegel Symmetry to the Spinning Particle Proper Time Supersymmetry, Phys. Lett. B 216 (1989) 302 [INSPIRE].

[9] M. Tonin, World sheet supersymmetric formulations of Green-Schwarz superstrings, Phys. Lett. B 266 (1991) 312 [INSPIRE].

[10] N. Berkovits, The Heterotic Green-Schwarz superstring on an $N=(2,0)$ superworldsheet, Nucl. Phys. B 379 (1992) 96 [hep-th/9201004] [INSPIRE].

[11] F. Delduc, A. Galperin, P.S. Howe and E. Sokatchev, A twistor formulation of the heterotic $D=10$ superstring with manifest $(8,0)$ world sheet supersymmetry, Phys. Rev. D 47 (1993) 578 [hep-th/9207050] [INSPIRE].

[12] A. Galperin and E. Sokatchev, A twistor formulation of the nonheterotic superstring with manifest world sheet supersymmetry, Phys. Rev. D 48 (1993) 4810 [hep-th/9304046] [INSPIRE].

[13] D.P. Sorokin, Superbranes and superembeddings, Phys. Rept. 329 (2000) 1 [hep-th/9906142] [INSPIRE].

[14] M. Matone, L. Mazzucato, I. Oda, D. Sorokin and M. Tonin, The superembedding origin of the Berkovits pure spinor covariant quantization of superstrings, Nucl. Phys. B 639 (2002) 182 [hep-th/0206104] [INSPIRE].

[15] E. Witten, Perturbative gauge theory as a string theory in twistor space, Commun. Math. Phys. 252 (2004) 189 [hep-th/0312171] [INSPIRE].

[16] N. Berkovits, An alternative string theory in twistor space for $N=4$ super Yang-Mills, Phys. Rev. Lett. 93 (2004) 011601 [hep-th/0402045] [INSPIRE].

[17] L. Mason and D. Skinner, Ambitwistor strings and the scattering equations, JHEP 07 (2014) 048 [arXiv: 1311.2564] [INSPIRE].

[18] N. Berkovits, Infinite Tension Limit of the Pure Spinor Superstring, JHEP 03 (2014) 017 [arXiv: 1311.4156] [INSPIRE].

[19] D.J. Gross, J.A. Harvey, E.J. Martinec and R. Rohm, Heterotic String Theory. 1. The Free Heterotic String, Nucl. Phys. B 256 (1985) 253 [inSPIRE].

[20] I. Bakhmatov and N. Berkovits, Pure Spinor b-ghost in a Super-Maxwell Background, JHEP 11 (2013) 214 [arXiv: 1310.3379] [INSPIRE].

[21] K. Lee and W. Siegel, Simpler superstring scattering, JHEP 06 (2006) 046 [hep-th/0603218] [INSPIRE].

[22] D. Friedan, E.J. Martinec and S.H. Shenker, Conformal Invariance, Supersymmetry and String Theory, Nucl. Phys. B 271 (1986) 93 [InSPIRE].

[23] N. Berkovits and P.S. Howe, Ten-dimensional supergravity constraints from the pure spinor formalism for the superstring, Nucl. Phys. B 635 (2002) 75 [hep-th/0112160] [INSPIRE].

[24] L.P. Hughston, The Wave Equation in Even Dimensions, in Further Advances in Twistor Theory, vol. 1, Research Notes in Mathematics 231, Longman (1990), pg. 26-27.

[25] N. Berkovits and S.A. Cherkis, Higher-dimensional twistor transforms using pure spinors, JHEP 12 (2004) 049 [hep-th/0409243] [INSPIRE].

[26] N. Berkovits, M. Bershadsky, T. Hauer, S. Zhukov and B. Zwiebach, Superstring theory on $A d S_{2} \times S^{2}$ as a coset supermanifold, Nucl. Phys. B 567 (2000) 61 [hep-th/9907200] [INSPIRE]. 